\title{
On Versality for Unfoldings of Smooth Section Germs
}

\author{
By \\ Shyūichi IZUMIYA*
}

\section{§0. Introduction}

In this article, we consider unfoldings of smooth section germs. The unfolding, which is introduced by René Thom, is an important notion to describe any situations in which qualitative picture of the object with a change of the parameters on which the object depends. The idea is an analogue to the deformation theory of singularities of complex varieties for real smooth cases. Hence, it can be thought as smooth families of section germs.

It turns out in many cases the study of all possible unfoldings leads to that of a single one, from which all others can be obtained. Such an unfolding, in some sense the largest one, should give all the essentially distinct bifurcation with respect to given equivalence relation; it is the versal unfolding.

In recent years, "the versality theorem" for categories of unfoldings of smooth map germs relative to some equivalence relations have been proved ([3], [4], [8]). Now, we say that the versality theorem holds if the algebraic notion of "infinitesimal versality" is the sufficient condition of the notion of "versality". But as the category of unfoldings of smooth vector field germs relative to coordinate transformations, there are examples for which "the versality theorem" cannot hold ([1]).

In this paper, we will single out the class of categories of unfoldings of smooth section germs of smooth vector bundles relative to various equivalence relations for which "the versality theorem" hold.

As applications of the main theorem, we have "versality theorems" for

Communicated by N. Shimada, December 2, 1980.

* Department of Mathematics, Nara Women's University, Nara 630, Japan.

Partially supported by Grant-in-Aid for Encouragement of Young Scientist (No. 57034), Ministry of Education. 
categories of unfoldings of smooth map germs ([3], [4], [8]) and the category of $G$-unfoldings of $G$-invariant function germs relative to $G$-right equivalence (Theorem 4.6). These are theorems which are already proved by many authors. Since our proof is a bit of an extension of Mather's method, it is slightly different of those of [2], [3] and [8]. The main theorem unifies not only these theorems but also gives new informations about many other categories. For example, these are the category of $G$-unfoldings of equivariant map germs relative to $G$-contact equivalence (Theorem 4.7) and the category of unfoldings of smooth section germs which are solutions of a linear partially differential equation, (for example, harmonic function germs), relative to some equivalence relations. (Theorems 4.4 and 4.5).

In Section 1, we will construct the category of $\Lambda$-unfoldings of section germs relative to the equivalence relation which is given by a subpseudo-group of the pseudo-group of local smooth fibre bundle automorphisms. Our main theorem is Theorem B, which will be formulated in Section 1 and proved in Sections 2 and 3. In Section 4, we will give some applications of Theorem B.

In this paper, smooth means class $C^{\infty}$.

\section{§1. Formulations}

In this section, we will construct the category of $\Lambda$-unfoldings and formulate the main theorem.

Let $M$ be a smooth manifold. We consider a smooth vector bundle $E(M)$ over $M$ with the fibre $V(M)$ and the set $\Gamma_{E}^{\infty}(M)$ of local smooth sections of $E(M)$.

Let $\Gamma_{E}^{A}(M)$ be a subset of $\Gamma_{E}^{\infty}(M)$ and $\mathscr{D}(M)$ be the pseudo-group of local diffeomorphisms on $M$.

Definition 1.1. Let $\mathscr{G}_{E}(M)$ be a subpseudo-group of $\mathscr{D}(M) \times \mathscr{D}(E(M))$. Then, we say that $\mathscr{G}_{E}(M)$ is essential if for any $(h, H) \in \mathscr{G}_{E}(M), H$ covers $h$.

We now have a definition of $\mathscr{G}_{E}$-equivalence for germs of elements of $\Gamma_{E}^{A}(M)$ as follows.

Definition 1.2. Let $\sigma:(M, a) \rightarrow(E(M), b)$ and $\sigma^{\prime}:\left(M, a^{\prime}\right) \rightarrow\left(E(M), b^{\prime}\right)$ be germs of elements of $\Gamma_{E}^{\lambda}(M)$ at $a$ and $a^{\prime}$ respectively. We say that $\sigma$ and $\sigma^{\prime}$ are $\mathscr{G}_{E}$-equivalent (and we write $\sigma \sim_{\mathscr{F}_{L}} \sigma^{\prime}$ ) if there exists a germ $(h, H)$ of elements of 
$G_{E}(M)$ at $(a, b) \in M \times E(M)$ with $(h(a), H(b))=\left(a^{\prime}, b^{\prime}\right)$ such that $H \circ \sigma=\sigma^{\prime} \circ h$.

Since we shall only consider germs throughout this article, we assume that $M=\boldsymbol{R}^{n}$ and every germs are defined at the origin.

\section{Notations.}

$\Gamma_{E}^{\Lambda}(n)=\left\{\sigma \mid \sigma:\left(\boldsymbol{R}^{n}, 0\right) \rightarrow E\left(\boldsymbol{R}^{n}\right) ;\right.$ germ of elements of $\left.\Gamma_{E}^{\Lambda}\left(\boldsymbol{R}^{n}\right)\right\}$.

$\Gamma_{E}^{\Lambda}(n)_{0}=\left\{\sigma \in \Gamma_{E}^{A}(n) \mid \sigma(0)=(0,0) \in \boldsymbol{R}^{n} \times V\left(\boldsymbol{R}^{n}\right) \cong E\left(\boldsymbol{R}^{n}\right)\right\}$.

$\mathscr{G}_{E}(n)=\left\{(h, H) \mid(h, H)\right.$ is a germ of elements of $\mathscr{G}_{E}\left(\boldsymbol{R}^{n}\right)$ at $\left.(0,0) \in \boldsymbol{R}^{n} \times E\left(\boldsymbol{R}^{n}\right)\right\}$.

Definition 1.3. By an $r$-dimensional $\Lambda$-unfolding of $\sigma \in \Gamma_{L}^{A}(n)_{0}$ we mean a smooth map germ

$$
\Sigma:\left(\boldsymbol{R}^{n} \times \boldsymbol{R}^{r},(0,0)\right) \longrightarrow\left(E\left(\mathbb{R}^{n}\right), 0\right)
$$

such that

i) $\Sigma_{u} \in \Gamma_{E}^{\Lambda}(n)$ for any $u \in\left(\boldsymbol{R}^{r}, 0\right)$, (where we define $\Sigma_{u}(x)=\Sigma(x, u)$ and $u \in\left(\boldsymbol{R}^{r}, 0\right)$ means that $u$ is a point of $\boldsymbol{R}^{r}$ near the origin),

ii) $\Sigma_{0}=\sigma$.

Then we will construct the category of $\Lambda$-unfoldings.

Definition 1.4. Let $\Sigma:\left(\boldsymbol{R}^{n} \times \boldsymbol{R}^{r},(0,0)\right) \rightarrow\left(E\left(\boldsymbol{R}^{n}\right), 0\right)$ be a $\Lambda$-unfolding of $\sigma \in \Gamma_{E}^{A}(n)_{0}$ and $f:\left(\boldsymbol{R}^{s}, 0\right) \rightarrow\left(\boldsymbol{R}^{r}, 0\right)$ be a smooth map germ. We say that

$$
\Omega:\left(\boldsymbol{R}^{n} \times \boldsymbol{R}^{s},(0,0)\right) \longrightarrow\left(E\left(\boldsymbol{R}^{n}\right), 0\right)
$$

is an incluced $\Lambda$-unfolding from $\Sigma$ by $f$ if it is defined by

$$
\Omega(x, v)=\Sigma(x, f(v)) \text { for any }(x, v) \in\left(\boldsymbol{R}^{n} \times \boldsymbol{R}^{s},(0,0)\right) .
$$

We write $\Omega=f^{*} \Sigma$.

Definition 1.5. Two $\Lambda$-unfoldings $\Sigma, \Omega:\left(\mathbb{R}^{n} \times \mathbb{R}^{r},(0,0)\right) \rightarrow\left(E\left(\boldsymbol{R}^{n}\right), 0\right)$ of $\sigma \in \Gamma_{E}^{A}(n)_{0}$ are said to be $\mathscr{G}_{E^{-}}$equivalent when there exists a map germ

$$
(h, H):\left(\boldsymbol{R}^{r}, 0\right) \longrightarrow \mathscr{G}_{E}(n)
$$

with the following properties:

(i) $(h, H)(0)=1$,

(ii) $(\widetilde{h, H}):\left(\boldsymbol{R}^{n} \times E\left(\boldsymbol{R}^{n}\right) \times \boldsymbol{R}^{r},(0,0,0)\right) \longrightarrow\left(\mathbb{R}^{n} \times E\left(\boldsymbol{R}^{n}\right),(0,0)\right)$

defined by $(h, H)(x, y, u)=(h(u)(x), H(u)(y))$ for $(x, y, u) \in\left(\mathbb{R}^{n} \times\right.$ $\left.E\left(\boldsymbol{R}^{n}\right) \times \boldsymbol{R}^{r},(0,0,0)\right)$ is a smooth map germ,

such that $H(u) \circ \Sigma_{u}=\Omega_{u} \circ h(u)$. 
Definition 1.6. Let $\Sigma:\left(\mathbb{R}^{n} \times \boldsymbol{R}^{r},(0,0)\right) \rightarrow\left(E\left(\mathbb{R}^{n}\right), 0\right)$ and $\Omega:\left(\boldsymbol{R}^{n} \times \mathbb{R}^{s}\right.$, $(0,0)) \rightarrow\left(E\left(\boldsymbol{R}^{n}\right), 0\right)$ be $\Lambda$-unfoldings of $\sigma \in \Gamma \frac{\Lambda}{E}(n)_{0}$. A $\mathscr{G}_{E}$-morphism from $\Omega$ to $\Sigma$ is a pair $\Phi=((h, H), f)$ where $(h, H):\left(\mathbb{R}^{s}, 0\right) \rightarrow \mathscr{G}_{E}(n)$ and $f:\left(\mathbb{R}^{s}, 0\right) \rightarrow\left(\mathbb{R}^{r}, 0\right)$ are smooth map germs such that $f^{*} \Sigma$ and $\Omega$ are $\mathscr{G}_{E}$-equivalent by $(h, H)$. We write $\Phi: \Omega \rightarrow \Sigma$.

In the above way, we have constructed the category of $\Lambda$-unfoldings of a germ of elements in $\Gamma_{E}(M)$ relative to $\mathscr{G}_{E}$-equivalence. The versal $\Lambda$-unfolding is defined as follows.

Definition 1.7. Let $\Sigma:\left(\mathbb{R}^{n} \times \mathbb{R}^{r},(0,0)\right) \rightarrow\left(E\left(\boldsymbol{R}^{n}\right), 0\right)$ be a $\Lambda$-unfolding of $\sigma \in \Gamma_{E}^{\Lambda}(n)_{0}$. Then $\Sigma$ is said to be a versal $\Lambda$-unfolding of $\sigma$ relative to $\mathscr{G}_{E}$ when for any $\Lambda$-unfolding $\Omega$ of $\sigma$ there exists a $\mathscr{G}_{E}$-morphism $\Phi: \Omega \rightarrow \Sigma$.

Remark. A versal $\Lambda$-unfolding of $\sigma$ relative to $\mathscr{G}_{E}$ has every information as $\Lambda$-unfoldings of $\sigma$ relative to the $\mathscr{G}_{E}$-equivalence. Hence, if we study the bifurcation of singularities of $\sigma$ in $\Gamma_{E}^{\Lambda}(n)$ with respect to $\mathscr{G}_{E}$-equivalence, it is enough to seek for a versal $\Lambda$-unfolding.

Now we have the influential candidate which characterize the versal $\Lambda$ unfolding of $\sigma \in \Gamma_{E}^{\Lambda}(n)_{0}$ relative to $\mathscr{G}_{E}$.

We will formulate as follows: Let $\theta(n)$ be the set of germs of smooth vector fields at the origin of $\boldsymbol{R}^{n}$. We remark that for any $\xi \in \theta(n)$ there exists a unique smooth map germ $\phi:\left(\boldsymbol{R}^{n} \times(-\varepsilon, \varepsilon),(0,0)\right) \rightarrow\left(\boldsymbol{R}^{n}, 0\right)$ with $\phi_{t} \in \mathscr{D}(n)$ and $\phi_{0}=1$ such that $d /\left.d t\left(\phi_{t}\right)\right|_{t=0}=\xi$ by the existence theorem for ordinary differential equations. Now, we define the "tangent space" $\theta_{E}^{\mathscr{s}}(n)$ corresponding to $\mathscr{G}_{E}(n)$ as follows:

$$
\begin{aligned}
& \theta_{E}^{\mathscr{s}}(n)=\left\{(\xi, \eta) \in \theta(n) \times \theta(E(n))\left|\xi=\frac{d}{d t}\left(h_{t}\right)\right|_{t=0}\right. \text { and } \\
&\left.\eta=\left.\frac{d}{d t}\left(H_{t}\right)\right|_{t=0} \text { for }\left(h_{t}, H_{t}\right) \in \mathscr{G}_{E}(n) \text { and }\left(h_{0}, H_{0}\right)=1\right\} .
\end{aligned}
$$

In general $\theta_{E}^{s}(n)$ does not have any useful algebraic structure even for $\mathbb{R}$-linear space structure.

Let $C_{0}^{\infty}\left(\boldsymbol{R}^{n}, E\left(\boldsymbol{R}^{n}\right)\right)$ be the space of smooth map germs $\mathbb{R}^{n} \rightarrow E\left(\boldsymbol{R}^{n}\right)$ at the origin.

If $\sigma \in \Gamma_{E}^{\Lambda}(n)$, we let

$$
d \sigma:\left(T \boldsymbol{R}^{n}, \pi_{n}^{-1}(0)\right) \longrightarrow\left(T E\left(\boldsymbol{R}^{n}\right), \pi^{-1}(0)\right)
$$

denote the tangent map germ of $\sigma$, then the following diagram commutes: 


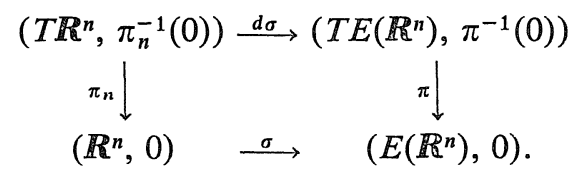

For any $\xi \in \theta(n)$, we can regard as $d \sigma \circ \xi \in C_{0}^{\infty}\left(\mathbb{R}^{n}, E\left(\mathbb{R}^{n}\right)\right)$, and for any $\eta \in \theta(E(n))$, we can also regard as $\eta \circ \sigma \in C_{0}^{\infty}\left(\mathbb{R}^{n}, E\left(\mathbb{R}^{n}\right)\right)$.

We define

$$
\widetilde{T}_{\sigma}: \theta(n) \times \theta(E(n)) \longrightarrow C_{0}^{\infty}\left(\mathbb{R}^{n}, E\left(\mathbb{R}^{n}\right)\right)
$$

by

$$
\tilde{T}_{\sigma}(\xi, \eta)=d \sigma \circ \xi+\eta \circ \sigma .
$$

Since $E\left(\mathbb{R}^{n}\right) \cong \mathbb{R}^{n} \times V\left(\mathbb{R}^{n}\right)$, we have a decomposition $C_{0}^{\infty}\left(\mathbb{R}^{n}, E\left(\mathbb{R}^{n}\right)\right) \cong C_{0}^{\infty}\left(\mathbb{R}^{n}, \mathbb{R}^{n}\right)$ $\oplus C_{0}^{\infty}\left(\mathbb{R}^{n}, V\left(\mathbb{R}^{n}\right)\right)$ and let $\pi_{V}: C_{0}^{\infty}\left(\mathbb{R}^{n}, E\left(\mathbb{R}^{n}\right)\right) \rightarrow C_{0}^{\infty}\left(\mathbb{R}^{n}, V\left(\mathbb{R}^{n}\right)\right)$ be the canonical projection. We let $T_{\sigma}=\pi_{V} \circ \widetilde{T}_{\sigma}$.

Definition 1.8. We say that

$$
T_{\sigma}^{\mathscr{G}}=T_{\sigma} \mid \theta_{E}^{\mathscr{G}}(n): \theta_{E}^{\mathscr{S}}(n) \longrightarrow C_{0}^{\infty}\left(\mathbb{R}^{n}, V\left(\mathbb{R}^{n}\right)\right)
$$

is an infinitesimal map of $\sigma$ relative to $\mathscr{G}_{E}$.

If $\theta_{E}^{\mathscr{S}}(n)$ is an $\mathbb{R}$-linear subspace of $\theta(n) \times \theta(E(n))$, the infinitesimal map of $\sigma$ is an $\mathbb{R}$-linear map.

We now assume that

Assumption (*). $\pi_{V}\left(\Gamma_{E}^{\Lambda}(n)\right)$ is an $\mathbb{R}$-affine subspace of $C_{0}^{\infty}\left(\mathbb{R}^{n}, V\left(\mathbb{R}^{n}\right)\right)$ (i.e. for any $\sigma, \omega \in \pi_{V}\left(\Gamma_{E}^{\Lambda}(n)\right)$, $\left.\{t \sigma+(1-t) \omega \mid t \in \mathbb{R}\} \subset \pi_{\Lambda}\left(\Gamma_{E}^{\Lambda}(n)\right)\right)$.

Remark. For any $\sigma \in \Gamma_{E}^{\Lambda}(n)$,

$$
\begin{array}{r}
C_{0}^{A}\left(\mathbb{R}^{n}, V\left(\mathbb{R}^{n}\right)\right)_{\sigma}=\left\{\begin{array}{r}
\tau \in C_{0}^{\infty}\left(\mathbb{R}^{n}, V\left(\mathbb{R}^{n}\right)\right)\left|\tau=\frac{d}{d t}\left(f_{t}\right)\right|_{t=0} \text { for } \\
f:\left(\mathbb{R}^{n} \times(-\varepsilon \varepsilon),(0,0)\right) \longrightarrow V\left(\mathbb{R}^{n}\right) \text { such that } \\
\left.f_{t} \in \pi_{V}\left(\Gamma_{E}^{A}(n)\right) \text { and } f_{0}=\pi_{V}(\sigma)\right\}
\end{array}\right.
\end{array}
$$

is an $\mathbb{R}$-linear subspace of $C_{0}^{\infty}\left(\mathbb{R}^{n}, V\left(\mathbb{R}^{n}\right)\right)$ which is independent of the choice of $\sigma$. Hence, we can denote it as $C_{0}^{\Lambda}\left(\mathbb{R}^{n}, V\left(\mathbb{R}^{n}\right)\right)$.

Now, we consider the inclusion map

$$
C_{0}^{\Lambda}\left(\mathbb{R}^{n}, V\left(\mathbb{R}^{n}\right)\right) \longrightarrow C_{0}^{\infty}\left(\mathbb{R}^{n}, V\left(\mathbb{R}^{n}\right)\right),
$$

then it induces the following injective linear map; 


$$
\begin{gathered}
C_{0}^{A}\left(\boldsymbol{R}^{n}, V\left(\boldsymbol{R}^{n}\right)\right) /\left(C_{0}^{A}\left(\boldsymbol{R}^{n}, V\left(\boldsymbol{R}^{n}\right)\right) \cap \mathrm{m}_{n} C_{0}^{\infty}\left(\boldsymbol{R}^{n}, V\left(\boldsymbol{R}^{n}\right)\right)\right) \longrightarrow \\
C_{0}^{\infty}\left(\boldsymbol{R}^{n}, V\left(\boldsymbol{R}^{n}\right)\right) / \mathfrak{m}_{n} C_{0}^{\infty}\left(\boldsymbol{R}^{n}, V\left(\boldsymbol{R}^{n}\right)\right)
\end{gathered}
$$

where $1 t_{n}$ denotes the maximal ideal of the ring of smooth function germs at $0 \in \boldsymbol{R}^{n}$. (cf. Definition 1.10).

Since $\operatorname{dim}_{\boldsymbol{R}}\left(C_{0}^{\infty}\left(\boldsymbol{R}^{n}, V\left(\boldsymbol{R}^{n}\right)\right) / \mathrm{m}_{n} C_{0}^{\infty}\left(\boldsymbol{R}^{n}, V\left(\boldsymbol{R}^{n}\right)\right)\right)=\operatorname{dim}_{\boldsymbol{R}} V\left(\boldsymbol{R}^{n}\right)$, there exist $a_{1}, \ldots, a_{s} \in C_{0}^{\Lambda}\left(\boldsymbol{R}^{n}, V\left(\boldsymbol{R}^{n}\right)\right)$ whose images are basis of $C_{0}^{A}\left(\boldsymbol{R}^{n}, V\left(\boldsymbol{R}^{n}\right)\right) /\left(C_{0}^{A}\left(\boldsymbol{R}^{n}, V\left(\boldsymbol{R}^{n}\right)\right) \cap m_{n} C_{0}^{\infty}\left(\boldsymbol{R}^{n}, V\left(\boldsymbol{R}^{n}\right)\right)\right)$ over $\boldsymbol{R}$.

Let $\Sigma:\left(\boldsymbol{R}^{n} \times \boldsymbol{R}^{r},(0,0)\right) \rightarrow\left(E\left(\boldsymbol{R}^{n}\right), 0\right)$ be a $\Lambda$-unfolding of $\sigma \in \Gamma_{E}^{\Lambda}(n)_{0}$, then $\partial \Sigma / \partial u_{i} \mid \boldsymbol{R}^{n} \times 0 \in C_{0}^{\Lambda}\left(\boldsymbol{R}^{n}, V\left(\boldsymbol{R}^{n}\right)\right)$. Hence, $V_{\Sigma}=\left\langle\partial \Sigma / \partial u_{i}\left|\boldsymbol{R}^{n} \times 0, \ldots, \partial \Sigma / \partial u_{i}\right| \boldsymbol{R}^{n} \times 0\right.$, $\left.a_{1}, \ldots, a_{s}\right\rangle_{\boldsymbol{R}}$ is an $\boldsymbol{R}$-linear subspace of $C_{0}^{\Lambda}\left(\boldsymbol{R}^{n}, V\left(\boldsymbol{R}^{n}\right)\right)$.

Definition 1.9. Let $\Sigma:\left(\boldsymbol{R}^{n} \times \boldsymbol{R}^{r},(0,0)\right) \rightarrow\left(E\left(\boldsymbol{R}^{n}\right), 0\right)$ be a $\Lambda$-unfolding of $\sigma \in \Gamma_{E}^{\Lambda}(n)_{0}$. Then $\Sigma$ is said to be an infinitesimally versal $\Lambda$-unfolding of $\sigma$ relative to $\mathscr{G}_{E}$ if

$$
C_{0}^{\Lambda}\left(\boldsymbol{R}^{n}, V\left(\boldsymbol{R}^{n}\right)\right) \subset T_{\sigma}^{\mathscr{G}}\left(\theta_{E}^{\mathscr{S}}(n)\right)+V_{\Sigma}
$$

If $\theta_{E}^{s}(n)$ is an $\boldsymbol{R}$-linear subspace of $\theta(n) \times \theta(E(n))$, then we have the following proposition.

Proposition A. Let $\sigma \in \Gamma_{E}^{A}(n)_{0}$ which satisfies the following condition:

$$
\operatorname{dim}_{\boldsymbol{R}}\left(C_{0}^{\Lambda}\left(\boldsymbol{R}^{n}, V\left(\boldsymbol{R}^{n}\right)\right)+T_{\sigma}^{\mathscr{G}}\left(\theta_{E}^{\mathscr{G}}(n)\right)\right) / T_{\sigma}^{\mathscr{G}}\left(\theta_{E}^{\mathscr{G}}(n)\right)<+\infty .
$$

If $\Sigma:\left(\boldsymbol{R}^{n} \times \boldsymbol{R}^{r},(0,0)\right) \rightarrow\left(E\left(\boldsymbol{R}^{n}\right), 0\right)$ is a versal $\Lambda$-unfolding of $\sigma$ relative to $\mathscr{G}_{E}$, then $\Sigma$ is infinitesimally versal.

Proof. Let $T_{\sigma}^{\mathscr{S}}\left(\theta_{E}^{\mathscr{S}}(n)\right)^{\Lambda}=T_{\sigma}^{\mathscr{G}}\left(\theta_{L}^{\mathscr{\varphi}}(n)\right) \cap C_{0}^{\Lambda}\left(\boldsymbol{R}^{n}, V\left(\boldsymbol{R}^{n}\right)\right)$, then $\operatorname{dim}_{\boldsymbol{R}} C_{0}^{A}\left(\boldsymbol{R}^{n}, V\left(\boldsymbol{R}^{n}\right)\right) / T_{\sigma}^{\mathscr{S}}\left(\theta_{E}^{\mathscr{S}}(n)\right)^{\Lambda}=\operatorname{dim}_{\boldsymbol{R}}\left(C_{0}^{\Lambda}\left(\boldsymbol{R}^{n}, V\left(\boldsymbol{R}^{n}\right)\right)+T_{\sigma}^{\mathscr{G}}\left(\theta_{E}^{\mathscr{S}}(n)\right)\right) / T_{\sigma}^{\mathscr{S}}\left(\theta_{E}^{\mathscr{S}}(n)\right)$ $=q<+\infty$.Hence, there exist $b_{1}, \ldots, b_{q} \in C_{0}^{\Lambda}\left(\boldsymbol{R}^{n}, V\left(\boldsymbol{R}^{n}\right)\right)$ such that $\left\{b_{1}\right\}, \ldots,\left\{b_{q}\right\}$ generate $C_{0}^{\Lambda}\left(\boldsymbol{R}^{n}, V\left(\boldsymbol{R}^{n}\right)\right) / T_{\sigma}^{\mathscr{G}}\left(\theta_{E}^{\mathscr{S}}(n)\right)^{\Lambda}$ over $\boldsymbol{R}$.

We now define

$$
\Omega:\left(\boldsymbol{R}^{n} \times \boldsymbol{R}^{q},(0,0)\right) \longrightarrow\left(E\left(\boldsymbol{R}^{n}\right), 0\right)
$$

by

$$
\Omega(x, v)=\left(x, \pi_{V}(\sigma)+v_{1} b_{1}(x)+\cdots+v_{q} b_{q}(x)\right)
$$

for any $(x, v) \in\left(\boldsymbol{R}^{n} \times \boldsymbol{R}^{q},(0,0)\right)$, then $\Omega$ is an infinitesimally versal $\Lambda$-unfolding of $\sigma$ relative to $\mathscr{G}_{E}$.

Since $\Sigma$ is a versal $\Lambda$-unfolding of $\sigma$ relative to $\mathscr{G}_{E}$, there exists a $\mathscr{G}_{E}$-morphism $\Phi: \Omega \rightarrow \Sigma$. Hence, by the direct computation and the definition of the 
infinitesimal versality, $\Sigma$ is an infinitesimally versal $\Lambda$-unfolding relative to $\mathscr{G}_{E}$.

Q.E.D.

In general, we say that "the versality theorem" holds if the converse of the above proposition holds.

We need a condition about the differential structure of $\mathbb{R}^{n}$ (i.e. local ring on $\boldsymbol{R}^{n}$ ) in order to characterize the category of $\Lambda$-unfoldings for which "the versality theorem" holds.

Let $C_{a}^{\infty}\left(\boldsymbol{R}^{n}\right)$ be the local $\boldsymbol{R}$-algebra consisting of smooth function germs at $a \in \boldsymbol{R}^{n}$. We denote $m_{a}^{\infty}\left(\boldsymbol{R}^{n}\right)$ the unique maximal ideal in $C_{a}^{\infty}\left(\boldsymbol{R}^{n}\right)$. For the convenience, we denote $\mathfrak{m}_{n}=\mathfrak{n}_{0}^{\infty}\left(\boldsymbol{R}^{n}\right)$.

Definition 1.10. Let $R(n)$ be a sub $\mathbb{R}$-algebra of $C_{0}^{\infty}\left(\boldsymbol{R}^{n}\right)$. Then $R(n)$ is said to be of finite type if there exist $p_{1}, \ldots, p_{k} \in R(n)$ such that $P^{*}\left(C_{a}^{\infty}\left(\boldsymbol{R}^{k}\right)\right)=R(n)$. Here, $P:\left(\boldsymbol{R}^{n}, 0\right) \rightarrow\left(\boldsymbol{R}^{k}, a\right)$ is defined by $P(x)=\left(p_{1}(x), \ldots, p_{k}(x)\right)$ and $P^{*}: C_{a}^{\infty}\left(\mathbb{R}^{h}\right)$ $\rightarrow C_{0}^{\infty}\left(\boldsymbol{R}^{n}\right)$ is defined by $P^{*}(h)=h \circ P$.

Now, we let $\mathscr{A u t}_{E}^{\infty}(M)$ be the pseudo-group consisting of local smooth fibre bundle automorphisms, then it is the maximum essential subpseudo-group of $\mathscr{D}(M) \times \mathscr{D}(E(M))$. We denote the "tangent space" corresponding to $\mathscr{A}_{\boldsymbol{u}}^{\infty}(M)$ by $\theta_{E}^{\infty}(n)$. Then $\theta_{E}^{\circ}(n)$ is naturally a $C_{0}^{\infty}\left(\boldsymbol{R}^{n}\right)$-module: the action of $C_{0}^{\infty}\left(\boldsymbol{R}^{n}\right)$ on $\theta_{E}^{\circ}(n)$ is defined by $h(X, X+Y)=(h X, h X+h Y)$ for $h \in C_{0}^{\infty}\left(\mathbb{R}^{n}\right)$ and $(X, X+Y) \in \theta_{E}^{\infty}(n)$. It is evident that $C_{0}^{\infty}\left(\boldsymbol{R}^{n}, V\left(\boldsymbol{R}^{n}\right)\right)$ is also a $C_{0}^{\infty}\left(\boldsymbol{R}^{n}\right)$-module. Because $R(n)$ is a sub $\boldsymbol{R}$-algebra of $C_{0}^{\infty}\left(\boldsymbol{R}^{n}\right), \theta_{E}^{*}(n)$ and $C_{0}^{\infty}\left(\boldsymbol{R}^{n}, V\left(\boldsymbol{R}^{n}\right)\right)$ are naturally $R(n)$-modules.

Definition 1.11. We say that a triple $\left(\Gamma_{E}^{A}(n), \mathscr{G}_{E}(n), R(n)\right)$ is essential if the following conditions hold:

(1) $\Gamma_{E}^{\Lambda}(n)$ satisfies Assumption (*),

(2) $\mathscr{G}_{E}\left(\boldsymbol{R}^{n}\right)$ is an essential subpseudo-group of $\mathscr{D}\left(\mathbb{R}^{n}\right) \times \mathscr{D}\left(E\left(\mathbb{R}^{n}\right)\right)$,

(3) $R(n)$ is a sub $\boldsymbol{R}$-algebra of $C_{0}^{\infty}\left(\boldsymbol{R}^{n}\right)$ of finite type such that

(a) $\left\langle C_{0}^{\Lambda}\left(\boldsymbol{R}^{n}, V\left(\boldsymbol{R}^{n}\right)\right)\right\rangle_{R(n)}$ is a finitely generated $R(n)$-module and $T_{\sigma}^{\mathscr{G}}\left(\theta_{E}^{\mathscr{S}}(n)\right)$ $\subset\left\langle C_{0}^{A}\left(\boldsymbol{R}^{n}, V\left(\boldsymbol{R}^{n}\right)\right)\right\rangle_{\boldsymbol{R}(n)}$ for any $\sigma \in \Gamma_{E}^{\Lambda}(n)$,

(b) $\theta_{E}^{s}(n)$ is a sub $R(n)$-module of $\theta_{E}^{o g}(n)$,

(c) the infinitesimal map $T_{\sigma}^{g}: \theta_{E}^{\xi}(n) \rightarrow\left\langle C_{0}^{A}\left(\boldsymbol{R}^{n}, V\left(\boldsymbol{R}^{n}\right)\right)\right\rangle_{R(n)}$ is an $R(n)$ homomorphism for any $\sigma \in \Gamma_{E}^{\Lambda}(n)$.

Then, our main theorem is the following.

Theorem B (The versality theorem). Let $\left(\Gamma_{E}^{A}(n), \mathscr{G}_{E}(n), R(n)\right)$ be the 
essential triple. Let $\sigma \in \Gamma_{E}^{\Lambda}(n)_{0}$ which satisfies the following condition:

$$
\operatorname{dim}_{\boldsymbol{R}}\left\langle C_{0}^{\Lambda}\left(\boldsymbol{R}^{n}, V\left(\boldsymbol{R}^{n}\right)\right)\right\rangle_{R(n)} /\left(C_{0}^{\Lambda}\left(\boldsymbol{R}^{n}, V\left(\boldsymbol{R}^{n}\right)\right)+T_{\sigma}^{\mathscr{G}}\left(\theta_{E}^{\mathscr{G}}(n)\right)\right)<+\infty .
$$

If $\Sigma$ is an infinitesimally versal $\Lambda$-unfolding of $\sigma$ relative to $\mathscr{G}_{E}$, then $\Sigma$ is versal relative to $\mathscr{G}_{E}$.

For the proof of Theorem B, it is enough to prove the following uniqueness theorem.

Theorem 1.12 (The uniqueness theorem). With the same hypotheses of Theorem $B$, if $\Sigma$ and $\Omega$ are infinitesimally versal $\Lambda$-unfoldings of $\sigma$ relative to $\mathscr{G}_{E}$ with the same dimension, then these are $\mathscr{G}_{E^{-i}}$ isomorphic.

If we assume the statement of Theorem 1.12, we can prove Theorem B as follows.

Proof of Theorem $B$. Let $\Sigma$ be an $r$-dimensional infinitesimally versal $\Lambda$ unfolding of $\sigma \in \Gamma_{E}^{\Lambda}(n)_{0}$ relative to $\mathscr{G}_{E}$. Let $\Omega$ be any $\Lambda$-unfolding of $\sigma$ whose dimension is $s$.

We now define

$$
\Omega^{*}:\left(\boldsymbol{R}^{n} \times \boldsymbol{R}^{r+s},(0,0)\right) \longrightarrow\left(E\left(\boldsymbol{R}^{n}\right), 0\right)
$$

by

$$
\Omega^{*}(x, u, v)=\left(x, \pi_{V}(\Omega)(x, v)-\pi_{V}(\sigma)+\pi_{V}(\Sigma)(x, u)\right)
$$

for any $(x, u, v) \in\left(\boldsymbol{R}^{n} \times \boldsymbol{R}^{r+s},(0,0)\right)$. Since $\pi_{V}\left(\Gamma_{E}^{\Lambda}(n)\right)$ is an $\boldsymbol{R}$-affine space, $\Omega^{*}$ is a $\Lambda$-unfolding of $\sigma$ and $\Omega$ is induced from $\Omega^{*}$ by the canonical inclusion $i$ : $\left(\boldsymbol{R}^{r}, 0\right) \rightarrow\left(\boldsymbol{R}^{r+s},(0,0)\right)$.

We also define

$$
\Sigma^{*}:\left(\boldsymbol{R}^{n} \times \boldsymbol{R}^{r+s},(0,0)\right) \longrightarrow\left(E\left(\boldsymbol{R}^{n}\right), 0\right)
$$

by

$$
\Sigma^{*}(x, u, v)=\Sigma(x, u)
$$

for any $(x, u, v) \in\left(\mathbb{R}^{n} \times \mathbb{R}^{r+s},(0,0)\right)$. Then $\Sigma^{*}$ is the $\Lambda$-unfolding of $\sigma$ which is induced from $\Sigma$ by the canonical projection.

Since $\Sigma$ is an infinitesimally versal $\Lambda$-unfolding of $\sigma$ relative to $\mathscr{G}_{E}, \Omega^{*}$ and $\Sigma^{*}$ are infinitesimally versal $\Lambda$-unfoldings of $\sigma$ relative to $\mathscr{G}_{E}$. Because $\Omega^{*}$ and $\Sigma^{*}$ have both $(r+s)$-dimension, these are $\mathscr{G}_{E}$-isomorphic by Theorem 1.12. Hence, there exists a $\mathscr{G}_{E}$-morphism from $\Omega$ to $\Sigma$. This completes the proof.

Q.E.D. 
We will prove Theorem 1.12 in Section 2 and Section 3.

\section{§2. Preliminaries}

In this section, we will prepare some tools in order to prove Theorem 1.12.

2-A) Ordinary differential equations.

Let $\Phi:\left(\mathbb{R}^{n} \times \mathbb{R}^{r} \times \mathbb{R},(0,0,0)\right) \rightarrow\left(E\left(\mathbb{R}^{n}\right), 0\right)$ be an $(r+1)$-dimensional $\Lambda$ unfolding of $\sigma \in \Gamma_{E}^{\Lambda}(n)_{0}$.

For any given elements $b_{1}, \ldots, b_{q} \in C_{0}^{A}\left(\mathbb{R}^{n}, V\left(\mathbb{R}^{n}\right)\right)$, let

$$
\begin{aligned}
X=\sum_{i=1}^{n} \xi_{i}(x, u, v, t & \frac{\partial}{\partial x_{i}}+\sum_{j=1}^{r} \eta_{j}(x, u, v, t) \frac{\partial}{\partial u_{i}} \\
& +\sum_{l=1}^{q} \tau_{l}(x, u, v, t) \frac{\partial}{\partial v_{l}}+\zeta(x, u, v, t) \frac{\partial}{\partial t}
\end{aligned}
$$

be a germ of vector fields on $\mathbb{R}^{n} \times \mathbb{R}^{r} \times \mathbb{R}^{s} \times \mathbb{R}$ at the origin. We now define

$$
\begin{aligned}
& \Phi^{\prime}(X)=\sum_{i=1}^{n} \xi_{i} \frac{\partial}{\partial x_{i}}+\sum_{k=1}^{s}\left(\sum_{i=1}^{n} \frac{\partial}{\partial x_{i}}\left(\Phi_{k}\right) \xi_{i}+\sum_{j=1}^{r} \frac{\partial}{\partial u_{j}}\left(\Phi_{k}\right) \eta_{j}\right. \\
& \left.\quad+\sum_{l=1}^{q} \tau_{l} b_{l}^{k}+\frac{\partial}{\partial t}\left(\Phi_{k}\right) \zeta\right) \frac{\partial}{\partial y_{k}}+\sum_{j=1}^{r} \eta_{j} \frac{\partial}{\partial u_{j}}+\sum_{l=1}^{q} \tau_{l} \frac{\partial}{\partial v_{l}}+\zeta \frac{\partial}{\partial t},
\end{aligned}
$$

where $\left(y_{1}, \ldots, y_{s}\right)$ is a coordinate of $V\left(\mathbb{R}^{n}\right)$ about the origin. Let

$$
\begin{gathered}
Y=\sum_{i=1}^{n} \xi_{i}^{\prime}(x, y, u, v, t) \frac{\partial}{\partial x_{i}}+\sum_{k=1}^{s} \lambda_{k}^{\prime}(x, y, u, v, t) \frac{\partial}{\partial y_{k}} \\
+\sum_{j=1}^{r} \eta_{j}^{\prime}(x, y, u, v, t) \frac{\partial}{\partial u_{j}}+\sum_{l=1}^{q} \tau_{l}^{\prime}(x, y, u, v, t) \frac{\partial}{\partial v_{l}} \\
+\zeta^{\prime}(x, y, u, v, t) \frac{\partial}{\partial t}
\end{gathered}
$$

be a germ of vector fields on $E\left(\mathbb{R}^{n}\right) \times \mathbb{R}^{r} \times \mathbb{R}^{a} \times \mathbb{R}$ at the origin. We also define

$$
\begin{aligned}
(Y \circ \Phi)^{\prime}=\sum_{i=1}^{n} \xi_{i}^{\prime} \frac{\partial}{\partial x_{i}}+\sum_{k=1}^{s}\left(\lambda_{k}^{\prime}(\Phi(x, u, t), u, v, t)+\sum_{l=1}^{q} \tau_{l}^{\prime} b_{l}^{k}\right) \frac{\partial}{\partial y_{k}} \\
+\sum_{j=1}^{r} \eta_{j}^{\prime} \frac{\partial}{\partial u_{j}}+\sum_{l=1}^{q} \tau_{l}^{\prime} \frac{\partial}{\partial v_{l}}+\zeta^{\prime} \frac{\partial}{\partial t}
\end{aligned}
$$

Lemma 2.1. Suppose there exist germs of vector fields $X$ on $\mathbb{R}^{n} \times \mathbb{R}^{r}$ $\times \mathbb{R}^{q} \times \mathbb{R}$ and $Y$ on $E\left(\mathbb{R}^{n}\right) \times \mathbb{R}^{r} \times \mathbb{R}^{q} \times \mathbb{R}$ at the origin such that the following conditions hold:

(1) $\Phi^{\prime}(X)=(Y \circ \Phi)^{\prime}$

(2) There exist $\quad \xi_{i} \in \mathrm{m}_{r} C_{0}^{\infty}\left(\mathbb{R}^{n+r+1}\right) \quad(i=1, \ldots, n), \eta_{j}, \tau_{l} \in \mathrm{m}_{r} C_{0}^{\infty}\left(\mathbb{R}^{n+r}\right)$ 
$(j=1, \ldots, r ; l=1, \ldots, q)$, and $\lambda_{k} \in \mathfrak{m}_{r} C_{0}^{\infty}\left(\boldsymbol{R}^{n+s+r+1}\right)(k=1, \ldots, s)$ such that

$$
X=\sum_{i=1}^{n} \xi_{i}(x, u, v) \frac{\partial}{\partial x_{i}}+\sum_{j=1}^{r} \eta_{j}(u, t) \frac{\partial}{\partial u_{j}}+\sum_{l=1}^{q} \tau_{l}(u, t) \frac{\partial}{\partial v_{l}}+\frac{\partial}{\partial t}
$$

and

$$
\begin{aligned}
Y & =\sum_{i=1}^{n} \xi_{i}(x, u, v) \frac{\partial}{\partial x_{i}}+\sum_{k=1}^{s} \lambda_{k}(x, y, u, t) \frac{\partial}{\partial y_{k}}+\sum_{j=1}^{r} \eta_{j}(u, t) \frac{\partial}{\partial u_{j}} \\
& +\sum_{l=1}^{q} \tau_{l}(u, t) \frac{\partial}{\partial v_{l}}+\frac{\partial}{\partial t} .
\end{aligned}
$$

(3) If we denote $X_{(u, t)}^{\prime}=\sum_{i=1}^{n} \xi_{i}(x, u, t) \partial / \partial x_{i}$ and $Y_{(u, t)}^{\prime}=\sum_{k=1}^{s} \lambda_{k}(x, y, u$, t) $\partial / \partial y_{k}$, then

$$
\left(X_{(u, t)}^{\prime}, X_{(u, t)}^{\prime}+Y_{(u, t)}^{\prime}\right) \in \theta_{E}^{\mathscr{g}}(n) \text { for any }(u, t) \in\left(\boldsymbol{R}^{r} \times \boldsymbol{R},(0,0)\right) .
$$

Then, $\Phi_{0}$ and $\Phi_{t}$ are $\mathscr{G}_{E}$-isomorphic for sufficiently small $t>0$.

Proof. Let $\phi_{t}(x, u, v, s)$ be the integral curve of $X$ on $\boldsymbol{R}^{n} \times \boldsymbol{R}^{r} \times \boldsymbol{R}^{q} \times \boldsymbol{R}$ which passes through $(x, u, v, s)$ at $t=0$.

Let $\psi_{t}(x, y, u, v, s)$ be the integral curve of $Y$ on $E\left(\boldsymbol{R}^{n}\right) \times \boldsymbol{R}^{r} \times \boldsymbol{R}^{q} \times \boldsymbol{R}$ which passes through $(x, y, u, v, s)$ at $t=0$.

Since both coefficient of $\partial / \partial t$ of $X$ and $Y$ are 1 , then

$$
\phi_{t}\left(\boldsymbol{R}^{n} \times \boldsymbol{R}^{r} \times \boldsymbol{R}^{q} \times\{0\}\right) \subset \boldsymbol{R}^{n} \times \boldsymbol{R}^{r} \times \boldsymbol{R}^{q} \times\{t\}
$$

and

$$
\psi_{t}\left(E\left(\boldsymbol{R}^{n}\right) \times \boldsymbol{R}^{r} \times \boldsymbol{R}^{q} \times\{0\}\right) \subset E\left(\boldsymbol{R}^{n}\right) \times \boldsymbol{R}^{r} \times \boldsymbol{R}^{q} \times\{t\}
$$

as germs. Hence,

$$
\phi_{t} \mid \boldsymbol{R}^{n} \times \boldsymbol{R}^{r} \times \boldsymbol{R}^{q} \times\{0\}: \boldsymbol{R}^{n} \times \boldsymbol{R}^{r} \times \boldsymbol{R}^{q} \times\{0\} \longrightarrow \boldsymbol{R}^{n} \times \boldsymbol{R}^{r} \times \boldsymbol{R}^{q} \times\{t\}
$$

and

$$
\psi_{t} \mid E\left(\boldsymbol{R}^{n}\right) \times \boldsymbol{R}^{r} \times \boldsymbol{R}^{q} \times\{0\}: E\left(\boldsymbol{R}^{n}\right) \times \boldsymbol{R}^{r} \times \boldsymbol{R}^{q} \times\{0\}
$$

are local diffeomorphisms for sufficiently small $t>0$.

By the condition (2), we can write

$$
\phi_{t}(x, u, v, 0)=(h(x, u, t), f(u, t), g(u, t), t) \text { and }
$$$$
\psi_{t}(x, y, u, v, 0)=((h(x, u, t), H(x, y, u, t)), f(u, t), g(u, v, t), t) .
$$

The relation $\Phi^{\prime}(X)=(Y \circ \Phi)^{\prime}$ guarantees that the following diagram commutes as germs: 


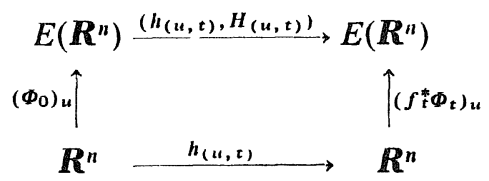

for any $(u, t) \in\left(\boldsymbol{R}^{r} \times \boldsymbol{R},(0,0)\right)$. By the definition of $\theta_{E}^{\varphi}(n)$, we can appreciate that $\left(h_{(u, t)}, H_{(u, t)}\right)$ is a family of elements of $\mathscr{G}_{E}(n)$.

Since $\xi_{i} \in m_{r} C_{0}^{\infty}\left(\boldsymbol{R}^{n+r+1}\right)$ and $\lambda_{k} \in m_{r} C_{0}^{\infty}\left(\boldsymbol{R}^{n+q+r+1}\right)$, we have

$$
h_{t}(x, 0)=x \text { and } H_{t}(x, y, 0)=(x, y) .
$$

Hence, $\left(\left(h_{t}, H_{t}\right), f_{t}\right)$ is the $\mathscr{G}_{E}$-isomorphism between $\Phi_{0}$ and $\Phi_{t}$. This completes the proof.

Q.E.D.

2-B) The local R-algebra $R(n)$.

Let $A_{a}^{n}$ be a sub $\boldsymbol{R}$-algebra of $C_{a}^{\infty}\left(\mathbb{R}^{n}\right)$ for any $n \in \mathbb{N}$. Let $f:\left(\mathbb{R}^{n}, a\right) \rightarrow$ $\left(\boldsymbol{R}^{p}, b\right)$ be a smooth map germ. We define an $\boldsymbol{R}$-algebra homomorphism

$$
f^{*}: C_{b}^{\infty}\left(\boldsymbol{R}^{p}\right) \longrightarrow C_{a}^{\infty}\left(\boldsymbol{R}^{n}\right)
$$

by

$$
f^{*}(h)=h \circ f \text {. }
$$

Definition 2.2. Let $f:\left(\boldsymbol{R}^{n}, a\right) \rightarrow\left(\boldsymbol{R}^{p}, b\right)$ be a smooth map germ such that $f^{*}\left(A_{b}^{p}\right) \subset A_{a}^{n}$. We say that $f^{*} A_{b}^{p} \rightarrow A_{a}^{n}$ has Property $(W)$ if the following holds:

Let $M$ be a finitely generated $A_{a}^{n}$-module. Suppose that $\operatorname{dim}_{\boldsymbol{R}} M / f^{*}\left(\mathfrak{m}_{b}^{\infty}\left(\boldsymbol{R}^{p}\right) \cap A_{b}^{p}\right) M<+\infty$, then $M$ is a finitely generated $A_{b}^{p}$-module via $f^{*}$.

For any non-negative integer $r$, we define

$$
C_{R(n)}^{\infty}\left(\boldsymbol{R}^{n+r}\right)=R(n) \otimes_{\mathbb{R}} C_{0}^{\infty}\left(\mathbb{R}^{r}\right) .
$$

Then, we can regard $C_{R(n)}^{\infty}\left(\boldsymbol{R}^{n+r}\right)$ as a sub $\boldsymbol{R}$-algebra of $C_{0}^{\infty}\left(\boldsymbol{R}^{n+r}\right)$. Let $\pi:\left(\boldsymbol{R}^{n+r},(0,0)\right) \rightarrow\left(\boldsymbol{R}^{r}, 0\right)$ be the canonical projection, then we have

$$
\pi^{*}\left(C_{0}^{\infty}\left(\boldsymbol{R}^{r}\right)\right) \subset C_{\boldsymbol{R}(n)}^{\infty}\left(\boldsymbol{R}^{n+r}\right) .
$$

The following lemma is "the preparation theorem" for $R(n)$.

Lemma 2.3. $\pi^{*}: C_{0}^{\infty}\left(\boldsymbol{R}^{r}\right) \rightarrow C_{\boldsymbol{R}(n)}^{\infty}\left(\boldsymbol{R}^{n+r}\right)$ has Property $(W)$.

Proof. Since $R(n)$ is of finite type, there exist $p_{1}, \ldots, p_{k} \in R(n)$ such that $P^{*}\left(C_{a}^{\infty}\left(\boldsymbol{R}^{k}\right)\right)=R(n)$. We now define

$$
\pi_{r}:\left(\boldsymbol{R}^{k+r},(0,0)\right) \longrightarrow\left(\boldsymbol{R}^{r}, 0\right)
$$

as the canonical projection. We also define 


$$
i: C_{0}^{\infty}\left(\boldsymbol{R}^{r}\right) \longrightarrow C_{0}^{\infty}\left(\boldsymbol{R}^{k}\right) \otimes_{\boldsymbol{R}} C_{0}^{\infty}\left(\boldsymbol{R}^{r}\right)
$$

by $i(f)=1 \otimes_{\mathbb{R}} f$. Then, we may consider that $\pi_{r}^{*}\left(\mathrm{~m}_{r}\right)=i\left(\mathrm{~m}_{r}\right)$ in $C_{(0,0)}^{\infty}\left(\mathbb{R}^{k+r}\right)$.

By Malgrange's preparation theorem (cf. Mather [5]), $\pi_{r}^{*}$ has Property (W). It follows that $i$ has also Property (W).

Since $P^{*}: C_{a}^{\infty}\left(\mathbb{R}^{k}\right) \rightarrow R(n)$ is surjective, then $P^{*} \otimes 1: C_{0}^{\infty}\left(\mathbb{R}^{k}\right) \otimes_{\mathbb{R}} C_{a}^{\infty}\left(\mathbb{R}^{r}\right)$ $\rightarrow C_{R(n)}^{\infty}\left(\boldsymbol{R}^{n+r}\right)$ is surjective. It has also Property (W).

It is clear that the composition of maps which have Property (W) has Property (W). Hence, $\pi^{*}=\left(P^{*} \otimes 1\right) \circ i$ has Property (W). This completes the proof.

Q.E.D.

Corollary. Let $M$ be a finitely generated $C_{R(n)}^{\infty}\left(\mathbb{R}^{n+r}\right)$-module. Let $N$ be a $C_{\mathbb{R}(n)}^{\infty}\left(\mathbb{R}^{n+r}\right)$-submodule of $M$ and $A$ be a finitely generated $C_{0}^{\infty}\left(\mathbb{R}^{r}\right)$-submodule via $\pi^{*}$.

Then

(a) $M=N+A+\pi^{*}\left(\mathrm{~m}_{r}\right) M$

implies

(b) $M=N+A$.

Proof. Let $M^{\prime}=M / N$ be the quotient $C_{R(n)}^{\infty}\left(\mathbb{R}^{n+r}\right)$-module and $P: M \rightarrow M^{\prime}$ be the canonical projection, then $M^{\prime}$ is a finitely generated $C_{R(n)}^{\infty}\left(\mathbb{R}^{n+r}\right)$-module. By the condition (a), we have $M^{\prime}=P(A)+\pi^{*}\left(\mathrm{~m}_{\mathrm{r}}\right) M^{\prime}$. Since $A$ is a finitely generated $C_{0}^{\infty}\left(\boldsymbol{R}^{r}\right)$-module, then $\operatorname{dim}_{\boldsymbol{R}} M^{\prime} / \pi^{*}\left(\mathrm{~m}_{r}\right) M^{\prime}<+\infty$. By Lemma 2.3, $M^{\prime}$ is a finitely generated $C_{0}^{\infty}\left(\mathbb{R}^{r}\right)$-module. Hence, we have $M^{\prime}=P(A)$ by Nakayama's lemma (cf. [5]). This completes the proof.

Q.E.D.

\section{§3. Proof of the Uniquness Theorem}

In order to prove Theorem 1.12, we need some lemmas.

Let $\Sigma, \Omega:\left(\mathbb{R}^{n} \times \mathbb{R}^{r},(0,0)\right) \rightarrow\left(E\left(\mathbb{R}^{n}\right), 0\right)$ be $\Lambda$-unfoldings of $\sigma \in \Gamma \frac{\Lambda}{E}(n)_{0}$. We define

$$
\Phi_{\Sigma, t}^{\Omega}:\left(\mathbb{R}^{n} \times \mathbb{R}^{r},(0,0)\right) \longrightarrow\left(E\left(\mathbb{R}^{n}\right), 0\right)
$$

by

$$
\Phi_{\Sigma, t}^{\Omega}(x, u)=\left(x, t \pi_{V}(\Omega)(x, u)+(1-t) \pi_{V}(\Sigma)(x, u)\right)
$$

for any $(x, u) \in\left(\mathbb{R}^{n} \times \mathbb{R}^{r},(0,0)\right)$ and $t \in[0,1]$. Since $\pi_{V}\left(\Gamma \frac{A}{E}(n)\right)$ is an $\mathbb{R}$-affine space, $\Phi_{\Sigma, t}^{\Omega}$ is a $\Lambda$-unfolding of $\sigma$ for any $t \in[0,1]$. 
Definition 3.1. In the above situation, suppose that $\Sigma$ and $\Omega$ are infinitesimally versal $\Lambda$-unfoldings of $\sigma$ relative to $\mathscr{G}_{E}$. We say that $\Sigma$ and $\Omega$ are linear versally homotopic relative to $\mathscr{G}_{E}$ if $\Phi_{\Omega, t}^{\Omega}$ are infinitesimally versal relative to $\mathscr{G}_{E}$ for any $t \in[0,1]$.

Lemma 3.2. For the proof of Theorem 1.12, it is enough to prove the statement of Theorem 1.12 for $\Lambda$-unfoldings $\Sigma$ and $\Omega$ which are linear versally homotopic relative to $\mathscr{G}_{E}$.

Proof. Let $\Sigma:\left(\mathbb{R}^{n} \times \mathbb{R}^{r},(0,0)\right) \rightarrow\left(E\left(\mathbb{R}^{n}\right), 0\right)$ be an infinitesimally versal $\Lambda$ unfolding of $\sigma$ relative to $\mathscr{G}_{E}$, then

$$
\begin{aligned}
C_{0}^{A}\left(\mathbb{R}^{n}, V\left(\mathbb{R}^{n}\right)\right)= & T_{\sigma}^{g}\left(\theta_{E}^{\mathscr{S}}(n)\right)^{\Lambda} \\
& +\left\langle\frac{\partial \Sigma}{\partial u_{1}}\left|\mathbb{R}^{n} \times 0, \ldots, \frac{\partial \Sigma}{\partial u_{r}}\right| \mathbb{R}^{n} \times 0, a_{1}, \ldots, a_{q}\right\rangle_{\mathbb{R}} .
\end{aligned}
$$

Since $\operatorname{dim}_{\mathbb{R}} C_{0}^{\infty}\left(\mathbb{R}^{n}, V\left(\mathbb{R}^{n}\right)\right) / T_{\sigma}^{\mathscr{g}}\left(\theta_{E}^{\mathscr{S}}(n)\right)^{\Lambda}=p \leqq r+q$, if necessary by the coordinate change, we may assume

$$
\begin{aligned}
C_{0}^{\Lambda}\left(\mathbb{R}^{n}, V\left(\mathbb{R}^{n}\right)\right) & =T_{\sigma}^{\mathscr{g}}\left(\theta_{E}^{\mathscr{S}}(n)\right)^{\Lambda} \\
& +\left\langle\frac{\partial \Sigma}{\partial u_{1}}\left|\mathbb{R}^{n} \times 0, \ldots, \frac{\partial \Sigma}{\partial u_{s}}\right| \mathbb{R}^{n} \times 0, a_{s+1}, \ldots, a_{p}\right\rangle_{\mathbb{R}} .
\end{aligned}
$$

We fix elements $c_{1}, \ldots, c_{p} \in C_{0}^{\Lambda}\left(\mathbb{R}^{n}, V\left(\mathbb{R}^{n}\right)\right)$ such that $\left\{c_{1}\right\}, \ldots,\left\{c_{p}\right\}$ are $\mathbb{R}^{2}-$ basis of $C_{0}^{\Lambda}\left(\mathbb{R}^{n}, V\left(\mathbb{R}^{n}\right)\right) / T_{\sigma}^{\mathscr{g}}\left(\theta_{E}^{\mathscr{s}}(n)\right)^{\Lambda}$. If necessary by the coordinate change, we may assume

$$
\begin{aligned}
& C_{0}^{\Lambda}\left(\mathbb{R}^{n}, V\left(\mathbb{R}^{n}\right)\right)=T_{\sigma}^{\mathscr{G}}\left(\theta_{E}^{\mathscr{G}}(n)\right)^{\Lambda} \\
& +\left\langle c_{1}, \frac{\partial \Sigma}{\partial u_{2}}\left|\mathbb{R}^{n} \times 0, \ldots, \frac{\partial \Sigma}{\partial u_{s}}\right| \mathbb{R}^{n} \times 0, a_{s+1}, \ldots, a_{p}\right\rangle_{\mathbb{R}} . \\
& C_{0}^{A}\left(\mathbb{R}^{n}, V\left(\mathbb{R}^{n}\right)\right)=T_{\sigma}^{\mathscr{G}}\left(\theta_{E}^{\mathscr{G}}(n)\right)^{\Lambda}+\left\langle c_{1}, c_{2}, \frac{\partial \Sigma}{\partial u_{3}} \mid \mathbb{R}^{n} \times 0, \ldots, a_{p}\right\rangle_{\mathbb{R}} . \\
& C_{0}^{\Lambda}\left(\mathbb{R}^{n}, V\left(\mathbb{R}^{n}\right)\right)=T_{\sigma}^{\mathscr{S}}\left(\theta_{E}^{\mathscr{S}}(n)\right)^{\Lambda}+\left\langle c_{1}, \ldots, c_{p-1}, a_{p}\right\rangle_{\mathbb{R}} .
\end{aligned}
$$

We now define

$$
\Sigma^{0}:\left(\mathbb{R}^{n} \times \mathbb{R}^{r},(0,0)\right) \longrightarrow\left(E\left(\mathbb{R}^{n}\right), 0\right)
$$

by

$$
\Sigma^{0}(x, u)=\left(x, \pi_{V}(\sigma)(x)+\sum_{i=1}^{s} u_{i}\left(\frac{\partial \Sigma}{\partial u_{i}} \mid \mathbb{R}^{n} \times 0\right)(x)+\sum_{i=s+1}^{p} u_{i} a_{i}(x)\right) .
$$

Since $\pi_{V}\left(\Gamma_{E}^{\Lambda}(n)\right)$ is an $\mathbb{R}$-affine space, $\Sigma^{0}$ is a $\Lambda$-unfolding of $\sigma$. Moreover, $\Sigma^{0}$ 
and $\Sigma$ are linear versally homotopic relative to $\mathscr{G}_{E}$.

Since $c_{1} \in C_{0}^{\Lambda}\left(\boldsymbol{R}^{n}, V\left(\boldsymbol{R}^{n}\right)\right)$, there exist $\xi \in T_{\sigma}^{\mathscr{G}}\left(\theta_{E}^{\mathscr{S}}(n)\right)^{\Lambda}$ and $\lambda_{i} \in \boldsymbol{R}(i=1, \ldots, p)$ such that $c_{1}=\sum_{i=1}^{s} \lambda_{i}\left(\partial \Sigma / \partial u_{i} \mid \boldsymbol{R}^{n} \times 0\right)+\sum_{i=s+1}^{p} \lambda_{l} a_{i}+\xi$. If $\lambda_{1}=0$, then $c_{1} \in\left\langle\partial \Sigma / \partial u_{2} \mid \boldsymbol{R}^{n} \times 0, \ldots, a_{p}\right\rangle_{\boldsymbol{R}}+T_{\sigma}^{\mathscr{S}}\left(\theta_{E}^{s}(n)\right)^{\Lambda}$. Hence, we have $C_{0}^{\Lambda}\left(\boldsymbol{R}^{n}, V\left(\boldsymbol{R}^{n}\right)\right)$ $=T_{\sigma}^{\mathscr{G}}(\theta(n))^{\Lambda}+\left\langle\partial \Sigma / \partial u_{2} \mid \boldsymbol{R}^{n} \times 0, \ldots, a_{p}\right\rangle_{\boldsymbol{R}}$. This contradicts to $\operatorname{dim}_{\boldsymbol{R}} C_{0}^{\Lambda}\left(\boldsymbol{R}^{n}, V\left(\boldsymbol{R}^{n}\right)\right) / T_{\sigma}^{g}(\theta(n))^{\Lambda}=p$. Hence, $\lambda_{1} \neq 0$ and let sign $\left(\lambda_{1}\right)$ $=\varepsilon_{1}$ (i.e. $\left.\varepsilon_{1}= \pm 1\right)$.

We also define

$$
\Sigma^{1}:\left(\boldsymbol{R}^{n} \times \boldsymbol{R}^{r},(0,0)\right) \longrightarrow\left(E\left(\boldsymbol{R}^{n}\right), 0\right)
$$

by

$$
\begin{aligned}
\Sigma^{1}(x, u)=\left(x, \pi_{V}(\sigma)(x)+\varepsilon_{1} u_{1} c_{1}(x)+\sum_{i=2}^{s} u_{i}(\right. & \left.\frac{\partial \Sigma}{\partial u_{i}} \mid \boldsymbol{R}^{n} \times 0\right)(x) \\
& \left.+\sum_{i=s+1}^{p} u_{i} a_{i}(x)\right) .
\end{aligned}
$$

Then $\Sigma^{1}$ is the $\Lambda$-unfolding of $\sigma$ which is linear versally homotopic to $\Sigma^{0}$ relative to $\mathscr{G}_{E}$.

By repeating this procedure, we can define $\Lambda$-unfoldings

$$
\Sigma^{j}:\left(\boldsymbol{R}^{n} \times \boldsymbol{R}^{r},(0,0)\right) \longrightarrow\left(E\left(\boldsymbol{R}^{n}\right), 0\right) \quad(j=1, \ldots, p)
$$

by

$$
\Sigma^{j}(x, u)=\left(x, \pi_{V}(\sigma)(x)+\sum_{i=1}^{j} \varepsilon_{i} u_{i} c_{i}(x)+\cdots\right) .
$$

such that $\Sigma^{j}$ and $\Sigma^{j-1}$ are linear versally homotopic relative to $\mathscr{G}_{E}$. In this situation, we say that $\Sigma$ is piecewise linear versally homotopic to $\Sigma^{p}$ relative to $\mathscr{G}_{E}$.

With the same argument, $\Omega$ is piecewise linear versally homotopic to $\Omega^{p}:\left(\boldsymbol{R}^{n} \times \boldsymbol{R}^{r},(0,0)\right) \rightarrow\left(E\left(\boldsymbol{R}^{n}\right), 0\right)$ which is defined by

$$
\Omega^{p}(x, u)=\left(x, \pi_{V}(\sigma)(x)+\sum_{i=1}^{p} \varepsilon_{i}^{\prime} u_{i} c_{i}(x)\right) .
$$

Then, there is a $\mathscr{G}_{E}$-isomorphism

$$
\Phi=(1, f): \Omega^{p} \cong \Sigma^{p}
$$

which is defined by

$$
f: \boldsymbol{R}^{r} \rightarrow \boldsymbol{R}^{r} ; f\left(u_{1}, \ldots, u_{r}\right)=\left(\left(\varepsilon_{1}^{\prime} / \varepsilon_{1}\right) u_{1}, \ldots,\left(\varepsilon_{p}^{\prime} / \varepsilon_{p}\right) u_{p}, u_{p+1}, \ldots, u_{r}\right)
$$

and $1 \in \mathscr{G}_{E}$. This completes the proof.

Q.E.D.

By the above lemma, we only consider the case that $\Sigma$ and $\Omega$ are linear versally homotopic $\Lambda$-unfoldings of $\sigma$. 
We now define

$$
\Phi:\left(\boldsymbol{R}^{n} \times \boldsymbol{R}^{r} \times \boldsymbol{R},(0,0,0)\right) \longrightarrow\left(E\left(\boldsymbol{R}^{n}\right), 0\right)
$$

by

$$
\Phi(x, u, t)=\left(x, \pi_{V}(\Phi, t)(x, u)\right) .
$$

Then $\Phi$ is an $(r+1)$-dimensional $\Lambda$-unfolding of $\sigma$ such that $\Phi(x, u, 0)=\Sigma(x, u)$ and $\Phi(x, u, 1)=\Omega(x, u)$.

We let

$$
\widetilde{C}_{0}^{\Lambda}\left(\mathbb{R}^{n+r+1}, V\left(\boldsymbol{R}^{n}\right)\right)=C_{R(n)}^{\infty}\left(\boldsymbol{R}^{n+r+1}\right) \otimes_{R(n)}\left\langle C_{0}^{\Lambda}\left(\boldsymbol{R}^{n}, V\left(\boldsymbol{R}^{n}\right)\right)\right\rangle_{R(n)} .
$$

It may be regarded as a $C_{\boldsymbol{R}(n)}^{\infty}\left(\boldsymbol{R}^{n+r+1}\right)$-submodule of $C_{0}^{\infty}\left(\boldsymbol{R}^{n}, V\left(\boldsymbol{R}^{n}\right)\right)$. Since $\left\langle C_{0}^{\Lambda}\left(\boldsymbol{R}^{n}, V\left(\boldsymbol{R}^{n}\right)\right)\right\rangle_{R(n)}$ is a finitely generated $R(n)$-module, then $\tilde{C}_{0}^{\Lambda}\left(\mathbb{R}^{n+r+1}, V\left(\mathbb{R}^{n}\right)\right)$ is finitely generated over $C_{R(n)}^{\infty}\left(\boldsymbol{R}^{n+r+1}\right)$.

We denote by $\tilde{\theta}(n+r+1) \times \tilde{\theta}(E(n)+r+1)$ the set of pairs $(X, Y)$ of smooth vector field germs along $\pi_{n}$ and $\pi_{E(n)}$ at origins respectively, where $\pi_{n}:\left(\boldsymbol{R}^{n}\right.$ $\left.\times \boldsymbol{R}^{r+1},(0,0)\right) \rightarrow\left(\boldsymbol{R}^{n}, 0\right)$ and $\pi_{E(n)}:\left(E\left(\boldsymbol{R}^{n}\right) \times \boldsymbol{R}^{r+1},(0,0)\right) \rightarrow\left(E\left(\boldsymbol{R}^{n}\right), 0\right)$ are canonical projections.

We also define

$$
\hat{\theta}_{E}^{s}(n+r+1)=C_{R(n)}^{\infty}\left(\boldsymbol{R}^{n+r+1}\right) \otimes_{R(n)} \theta_{E}^{s}(n),
$$

then it is a $C_{\boldsymbol{R}(n)}^{\infty}\left(\boldsymbol{R}^{n+r+1}\right)$-module, because $\theta_{E}^{\mathscr{s}}(n)$ has an $R(n)$-module structure. We may regard $\hat{\theta}_{E}^{s}(n+r+1)$ as a $C_{\boldsymbol{R}(n)}^{\infty}\left(\boldsymbol{R}^{n+r+1}\right)$-submodule of $\hat{\theta}(n+r+1) \times$ $\hat{\theta}(E(n)+r+1)$.

Next, we construct a homomorphism between these modules as follows:

$$
d_{x} \Phi: T \boldsymbol{R}^{n} \times \boldsymbol{R}^{r+1} \longrightarrow T E\left(\boldsymbol{R}^{n}\right)
$$

by

$$
d_{x} \Phi\left(\left(x_{0}, v\right),(u, t)\right)=d\left(\Phi_{(u, t)}\right)_{x_{0}}(v)
$$

Let

$$
\begin{aligned}
\tilde{\pi}_{V} & : C_{0}^{\infty}\left(\boldsymbol{R}^{n+r+1}, T E\left(\boldsymbol{R}^{n}\right)\right)=C_{0}^{\infty}\left(\boldsymbol{R}^{n+r+1}, E\left(\boldsymbol{R}^{n}\right) \times E\left(\boldsymbol{R}^{n}\right)\right) \\
& =C_{0}^{\infty}\left(\boldsymbol{R}^{n+r+1}, E\left(\boldsymbol{R}^{n}\right) \times \boldsymbol{R}^{n} \times V\left(\boldsymbol{R}^{n}\right)\right) \longrightarrow C_{0}^{\infty}\left(\mathbb{R}^{n+r+1}, V\left(\mathbb{R}^{n}\right)\right)
\end{aligned}
$$

be the canonical projection. We define

$$
T_{\Phi}: \hat{\theta}(n+r+1) \times \hat{\theta}(E(n)+r+1) \longrightarrow C_{0}^{\infty}\left(\boldsymbol{R}^{n+r+1}, V\left(\boldsymbol{R}^{n}\right)\right)
$$

by

$$
T_{\Phi}(X, Y)=\tilde{\pi}\left(d_{x} \Phi \circ\left(X, \mathrm{id}_{R^{r+1}}\right)+Y \circ\left(\Phi, \mathrm{id}_{R^{r+1}}\right)\right) .
$$


See the following diagram:

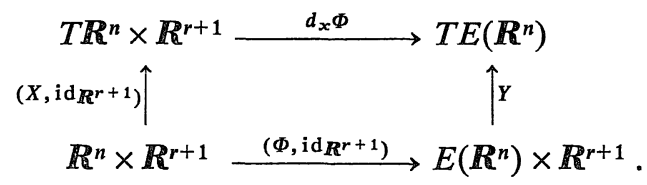

Then, we define a $C_{R(n)}^{\infty}\left(\mathbb{R}^{n+r+1}\right)$-homomorphism

$$
T_{\Phi}^{\mathscr{g}}: \hat{\theta}_{E}^{\mathscr{S}}(n+r+1) \longrightarrow \widetilde{C}_{0}^{\Lambda}\left(\mathbb{R}^{n+\boldsymbol{r}+1}, V\left(\boldsymbol{R}^{n}\right)\right)
$$

by

$$
T_{\Phi}^{\mathscr{G}}=T_{\Phi} \mid \tilde{\theta}_{E}^{\mathscr{G}}(n+r+t) .
$$

Since $\operatorname{dim}_{\boldsymbol{R}}\left\langle C_{0}^{\Lambda}\left(\boldsymbol{R}^{n}, V\left(\mathbb{R}^{n}\right)\right)\right\rangle_{R(n)} /\left(C_{0}^{\Lambda}\left(\mathbb{R}^{n}, V\left(\mathbb{R}^{n}\right)\right)+T_{\sigma}^{\mathscr{g}}\left(\theta_{E}^{\leftrightarrow}(n)\right)\right)<+\infty$, there exist $b_{1}, \ldots, b_{s} \in\left\langle C_{0}^{\Lambda}\left(\mathbb{R}^{n}, V\left(\mathbb{R}^{n}\right)\right)\right\rangle_{R(n)}$ such that

$(\#)\left\langle C_{0}^{\Lambda}\left(\mathbb{R}^{n}, V\left(\mathbb{R}^{n}\right)\right)\right\rangle_{R(n)}=C_{0}^{\Lambda}\left(\mathbb{R}^{n}, V\left(\mathbb{R}^{n}\right)\right)+T_{\sigma}^{\mathscr{g}}\left(\theta_{E}^{\mathscr{G}}(n)\right)+\left\langle b_{1}, \ldots, b_{s}\right\rangle_{\mathbb{R}}$.

Lemma 3.3. $\mathfrak{m}_{r} \widetilde{C}_{0}^{\Lambda}\left(\mathbb{R}^{n+r+1}, V\left(\boldsymbol{R}^{n}\right)\right)=\mathfrak{m}_{r} T_{\Phi}^{\varphi}\left(\tilde{\theta}_{E}^{g}(n+r+1)\right)$

$$
+\left\langle\frac{\partial \Phi}{\partial u_{1}}, \ldots, \frac{\partial \Phi}{\partial u_{r}}, a_{1}, \ldots, a_{q}, b_{1}, \ldots b_{s}\right\rangle_{\mathrm{nt}_{r} C_{0}^{\infty}\left(R^{r+1}\right)} \text {. }
$$

Proof. Since $\Sigma$ is infinitesimally versal relative to $\mathscr{G}_{E}$,

$$
C_{0}^{\Lambda}\left(\boldsymbol{R}^{n}, V\left(\mathbb{R}^{n}\right)\right) \subset T_{\sigma}^{\mathscr{g}}\left(\theta_{E}^{g}(n)\right)+V_{\Sigma} .
$$

Hence, by the formula (\#), we have

$$
\left\langle C_{0}^{\Lambda}\left(\boldsymbol{R}^{n}, V\left(\boldsymbol{R}^{n}\right)\right)\right\rangle_{R(n)}=T_{\sigma}^{\mathscr{g}}\left(\theta_{E}^{\mathscr{g}}(n)\right)+V_{\Sigma}+\left\langle b_{1}, \ldots, b_{s}\right\rangle_{\boldsymbol{R}} .
$$

Let $h \in \widetilde{C}_{0}^{\Lambda}\left(\boldsymbol{R}^{n+r+1}, V\left(\mathbb{R}^{n}\right)\right)$, then $h \mid \mathbb{R}^{n} \times 0 \in\left\langle C_{0}^{\Lambda}\left(\boldsymbol{R}^{n}, V\left(\boldsymbol{R}^{n}\right)\right)\right\rangle_{R(n)}$ and there exist $(X, Y) \in \theta_{E}^{s}(n), \xi_{i}, \eta_{j}, \mu_{l} \in \mathbb{R}(i=1, \ldots, r ; j=1, \ldots, s ; l=1, \ldots, q)$ such that

$$
h\left|\boldsymbol{R}^{n} \times 0=T_{\sigma}^{\mathscr{G}}(X, Y)+\sum_{i=1}^{r} \xi_{i} \frac{\partial \Sigma}{\partial u_{i}}\right| \boldsymbol{R}^{n} \times 0+\sum_{l=1}^{q} \mu_{l} a_{l}+\sum_{j=1}^{s_{j}} \eta_{j} b_{j} .
$$

We now let

$$
h^{\prime}=h-T_{\Phi}^{\mathscr{g}}(X, Y)-\sum_{i=1}^{r} \xi_{i} \frac{\partial \Sigma}{\partial u_{i}}-\sum_{l=1}^{q} \mu_{l} a_{l}-\sum_{j=1}^{s} \eta_{j} b_{j},
$$

then $h^{\prime} \in \widetilde{C}_{0}^{A}\left(\boldsymbol{R}^{n+\boldsymbol{r}+1}, V\left(\boldsymbol{R}^{n}\right)\right)$ and $h^{\prime} \mid \boldsymbol{R}^{n} \times 0=0$. Hence, by the Hadamard's lemma, we have the following:

$$
\begin{aligned}
& \tilde{C}_{0}^{A}\left(\boldsymbol{R}^{n+r+1}, V\left(\boldsymbol{R}^{n}\right)\right)=T_{\Phi}^{\mathscr{S}}\left(\tilde{\theta}_{E}^{S}(n+r+1)\right) \\
& \quad+\left\langle\frac{\partial \Phi}{\partial u_{1}}, \ldots, \frac{\partial \Phi}{\partial u_{r}}, a_{1}, \ldots, a_{q}, b_{1}, \ldots, b_{s}\right\rangle_{\boldsymbol{R}}+\mathfrak{m}_{r+1} \tilde{C}_{0}^{\Lambda}\left(\boldsymbol{R}^{n+r+1}, V(\boldsymbol{R})\right) .
\end{aligned}
$$


Then,

$$
\begin{aligned}
& \widetilde{C}_{0}^{\Lambda}\left(\mathbb{R}^{n+r+1}, V\left(\mathbb{R}^{n}\right)\right)=T_{\Phi}^{\mathscr{S}}\left(\tilde{\theta}_{E}^{s}(n+r+1)\right) \\
& \quad+\left\langle\frac{\partial \Phi}{\partial u_{1}}, \ldots, \frac{\partial \Phi}{\partial u_{r}}, a_{1}, \ldots a_{q}, b_{1}, \ldots, b_{s}\right\rangle_{C_{0}^{\infty}\left(\mathbb{R}^{r+1}\right)}+\mathrm{m}_{r+1} \tilde{C}_{0}^{\Lambda}\left(\mathbb{R}^{n+r+1}, V\left(\mathbb{R}^{n}\right)\right) .
\end{aligned}
$$

Hence, we can apply the corollary of Lemma 2.3 to this situation. Then, we have the following:

$$
\begin{aligned}
\widetilde{C}_{0}^{\Lambda}\left(\mathbb{R}^{n+r+1}, V\left(\mathbb{R}^{n}\right)\right) & =T_{\Phi}^{\wp}\left(\theta_{E}^{\wp}(n+r+1)\right) \\
& +\left\langle\frac{\partial \Phi}{\partial u_{1}}, \ldots, \frac{\partial \Phi}{\partial u_{r}}, a_{1}, \ldots, a_{q}, b_{1}, \ldots, b_{s}\right\rangle C_{0}^{\infty}\left(\mathbb{R}^{r+1}\right) .
\end{aligned}
$$

This completes the proof.

Q.E.D.

We now have tools to prove Theorem 1.12.

Proof of Theorem 1.12. Because $[0,1]$ is compact and $\Phi$ is linear versal homotopy between $\Sigma$ and $\Omega$, it is enough to prove $\Phi_{0}$ and $\Phi_{t}$ are $\mathscr{G}_{E}$-isomorphic for sufficiently small $t>0$.

Since $\Phi(x, 0, t)=\sigma(x)$, then $\partial \Phi(x, 0, t) / \partial t=0 . \quad$ Hence, $\partial \Phi / \partial t \in \mathfrak{m}_{r} \widetilde{C}_{0}^{\Lambda}\left(\mathbb{R}^{n+r+1}\right.$, $\left.V\left(\mathbb{R}^{n}\right)\right)$, then there exist $\left(X_{l}, Y_{l}\right) \in \tilde{\theta}_{E}^{s}(n+r+1), \xi_{l}^{\prime} \in \mathrm{m}_{r}(l=1, \ldots, m)$ and $\eta_{j}, \zeta_{k}$ $\in \mathrm{m}_{r} C_{0}^{\infty}\left(\mathbb{R}^{r+1}\right)(j=1, \ldots, r ; k=1, \ldots, s+q)$ such that

$$
\frac{\partial \Phi}{\partial t}=\sum_{l=1}^{m} \xi_{l}^{\prime} T_{\Phi}\left(X_{l}, Y_{l}\right)+\sum_{j=1}^{r} \eta_{j} \frac{\partial \Phi}{\partial u_{j}}+\sum_{k=1}^{s} \zeta_{k} b_{k}+\sum_{k=s+1}^{s+q} \zeta_{k} a_{k-s} .
$$

There are $\kappa_{i}^{l} \in C_{0}^{\infty}\left(\mathbb{R}^{n+r+1}\right)$ and $\tau_{\iota}^{l} \in C_{0}^{\infty}\left(\mathbb{R}^{n+q^{\prime}+r+1}\right)\left(i=1, \ldots, n ; \iota=1, \ldots, q^{\prime}\right)$ such that

$$
X_{l}=\sum_{i=1}^{n} \kappa_{i}^{l} \frac{\partial}{\partial x_{i}}
$$

and

$$
Y_{l}=\sum_{i=1}^{n} \kappa_{i}^{l} \frac{\partial}{\partial x_{i}}+\sum_{\iota=1}^{q^{\prime}} \tau_{\iota}^{l} \frac{\partial}{\partial y_{\iota}},
$$

where $\left(y_{1}, \ldots, y_{q^{\prime}}\right)$ denotes a coordinate of $V\left(\mathbb{R}^{n}\right)$ about the origin.

Let $\xi_{i}=\sum_{l=1}^{m} \xi_{l}^{\prime} \kappa_{i}^{l}$ and $\lambda_{\iota}=\sum_{l=1}^{m} \xi_{l}^{\prime} \tau_{i}^{l}$, then $\xi_{i} \in \mathfrak{m}_{r} C_{0}^{\infty}\left(\mathbb{R}^{n+r+1}\right), \lambda_{\iota} \in$ $m_{r} C_{0}^{\infty}\left(\mathbb{R}^{n+q^{\prime}+r+1}\right)$ and

$$
\begin{aligned}
\frac{\partial \Phi}{\partial t}= & T_{\sigma}^{g}\left(\sum_{i=1}^{n} \xi_{i} \frac{\partial}{\partial x_{i}}, \sum_{i=1}^{n} \xi_{i} \frac{\partial}{\partial x_{i}}+\sum_{\imath=1}^{q^{\prime}} \lambda_{\iota} \frac{\partial}{\partial y_{\iota}}\right) \\
& +\sum_{j=1}^{r} \eta_{j} \frac{\partial \Phi}{\partial u_{j}}+\sum_{k=1}^{q} \zeta_{k} b_{k}+\sum_{k=s+1}^{s+q} \zeta_{k} a_{k-s} .
\end{aligned}
$$

We now define 


$$
X=\sum_{i=1}^{n}\left(-\xi_{i}\right) \frac{\partial}{\partial x_{i}}+\sum_{j=1}^{r}\left(-\eta_{j}\right) \frac{\partial}{\partial u_{j}}+\sum_{k=1}^{s+q}\left(-\zeta_{k}\right) \frac{\partial}{\partial v_{k}}+\frac{\partial}{\partial t}
$$

and

$$
\begin{aligned}
Y & =\sum_{i=1}^{n}\left(-\xi_{i}\right) \frac{\partial}{\partial x_{i}}+\sum_{\imath=1}^{q^{\prime}} \lambda_{\iota} \frac{\partial}{\partial y_{\iota}}+\sum_{j=1}^{r}\left(-\eta_{j}\right) \frac{\partial}{\partial u_{j}} \\
& +\sum_{k=1}^{s+q}\left(-\zeta_{k}\right) \frac{\partial}{\partial v_{k}}+\frac{\partial}{\partial t},
\end{aligned}
$$

then these are vector field germs at origins which satisfy the conditions of Lemma 2.1 .

Hence, $\Phi_{0}$ and $\Phi_{t}$ are $\mathscr{G}_{E}$-isomorphic for sufficiently small $t>0$. This completes the proof.

Q.E.D.

\section{§4. Applications}

Here, we give some applications of Theorem B.

A) The case $R(n)=C_{0}^{\infty}\left(\boldsymbol{R}^{n}\right)$.

A-1) $\quad E(M)=M \times \boldsymbol{R}^{p}$.

A-1-a) We let

$\mathscr{G}_{E}(M)=\mathscr{R}(M)=\{(h, h \times L) \mid h$ is a local diffeomorphism on $M$ and $L$ is a parallel transformation on $\left.\boldsymbol{R}^{p}\right\}$

and $\Gamma_{E}^{\Lambda}(M)=\Gamma_{E}^{\infty}(M)$, then $\mathscr{G}_{E}$-equivalence of elements of $\Gamma_{E}^{\Lambda}(n)$ is exactly the right equivalence of smooth map germs $\left(\boldsymbol{R}^{n}, 0\right) \rightarrow\left(\boldsymbol{R}^{p}, 0\right)$.

It is clear that the infinitesimal map of a smooth map germ $f:\left(\boldsymbol{R}^{n}, 0\right) \rightarrow$ $\left(\boldsymbol{R}^{p}, 0\right)$ relative to $\mathscr{R}$ is given by the differential map of $f$ :

$$
d f: \theta(n) \longrightarrow C_{0}^{\infty}\left(\boldsymbol{R}^{n}, \boldsymbol{R}^{p}\right) .
$$

The image of $d f$ is the $C_{0}^{\infty}\left(\boldsymbol{R}^{n}\right)$-submodule of $C_{0}^{\infty}\left(\boldsymbol{R}^{n}, \boldsymbol{R}^{p}\right)$ which is generated by $\left(\partial f / \partial x_{i}(i=1, \ldots, n)\right.$. We write it $J(f)$ and call it Jacobian module of $f$. Hence, $\left(C_{0}^{\infty}\left(\boldsymbol{R}^{n}, \boldsymbol{R}^{p}\right), \mathscr{R}(n), C_{0}^{\infty}\left(\boldsymbol{R}^{n}\right)\right)$ is essential.

A $\Lambda$-unfolding of $f$ is the ordinary unfolding which has defined by Mather ([4]). Then we have the following theorem.

Theorem 4.1 (Thom-Mather-Zakalyukin [4], [8]). Let $F:\left(\boldsymbol{R}^{n} \times \boldsymbol{R}^{r},(0,0)\right)$ $\rightarrow\left(\boldsymbol{R}^{p}, 0\right)$ be an unfolding of smooth germ $f:\left(\boldsymbol{R}^{n}, 0\right) \rightarrow\left(\boldsymbol{R}^{p}, 0\right)$.

If

$$
C_{0}^{\infty}\left(\boldsymbol{R}^{n}, \boldsymbol{R}^{p}\right)=J(f)+V_{F},
$$

then $F$ is a versal unfolding of $f$ relative to $\mathscr{R}$. 
A-1-b) We let

$\mathscr{G}_{E}(M)=\mathscr{A}_{u} t_{E}^{V}(M)=\{(h, H) \mid H$ is a local bundle automorphism which covers $h$.

and $\Gamma_{E}^{A}(M)=\Gamma_{E}^{\propto}(M)$, then the $\mathscr{G}_{E}$-equivalence of elements of $\Gamma_{E}^{A}(n)$ is the $\mathscr{K}$ equivalence of smooth map germ $\left(\boldsymbol{R}^{n}, 0\right) \rightarrow\left(\boldsymbol{R}^{n}, 0\right)$ which has been introduced by Mather. (See Section 2 in [5]. The definition of the $\mathscr{K}$-equivalence is not given as above, but it has been implicitly shown in [5] that $\mathscr{K}$-equivalence is equivalent to the above $\mathscr{A}_{u} E_{E}^{V}$-equivalence.) By this reason, we write $\mathscr{A}_{u t}^{V}(n)$ $=\mathscr{K}(n, p)$.

The triple $\left(C_{0}^{\infty}\left(\boldsymbol{R}^{n}, \boldsymbol{R}^{p}\right), \mathscr{K}(n, p), C_{0}^{\infty}\left(\boldsymbol{R}^{n}\right)\right)$ is clearly essential.

By the easy calculation, it is proved that the image of the infinitcsimal map of $f:\left(\boldsymbol{R}^{n}, 0\right) \rightarrow\left(\mathbb{R}^{p}, 0\right)$ relative to $\mathscr{K}(n, p)$ is given by

$$
J(f)+f^{*}\left(\mathrm{~m}_{p}\right) C_{0}^{\infty}\left(\boldsymbol{R}^{n}, \mathbb{R}^{p}\right) .
$$

Theorem 4.2 (Martinet [3], Golubitsky-Schaeffer [2]). Let $F:\left(\mathbb{R}^{n} \times \boldsymbol{R}^{r}\right.$, $(0,0) \rightarrow\left(\boldsymbol{R}^{p}, 0\right)$ be an unfolding of a smooth map germ $f:\left(\mathbb{R}^{n}, 0\right) \rightarrow\left(\boldsymbol{R}^{p}, 0\right)$. If

$$
C_{0}^{\infty}\left(\boldsymbol{R}^{n}, \boldsymbol{R}^{p}\right)=J(f)+f^{*}\left(m_{p}\right) C_{0}^{\infty}\left(\mathbb{R}^{n}, \mathbb{R}^{p}\right)+V_{F},
$$

then $F$ is a versal unfolding of $f$ relative to $\mathscr{K}$.

A-2) $\quad E\left(\boldsymbol{R}^{n}\right)=\boldsymbol{R}^{n} \times \boldsymbol{R}^{n}$.

Let $\mathscr{G}_{E}\left(\boldsymbol{R}^{n}\right)=\mathscr{C}\left(\boldsymbol{R}^{n}\right)=\left\{(h, h \times h) \mid h\right.$ is a local diffeomorphism on $\left.\mathbb{R}^{n}\right\}$ and $\Gamma_{E}^{A}\left(\boldsymbol{R}^{n}\right)=\Gamma_{E}^{\infty}\left(\boldsymbol{R}^{n}\right)$, then two elements $f, g \in \Gamma_{E}^{\infty}(n)_{0}$ is $\mathscr{C}$-equivalent if and only if there exists invertible germ $h:\left(\boldsymbol{R}^{n}, 0\right) \rightarrow\left(\boldsymbol{R}^{n}, 0\right)$ such that $h \circ f=g \circ h$.

In this case, $\theta_{E}^{\mathscr{S}}(n)=\theta(n)$ and the infinitesimal map

$$
T_{f}: \theta(n) \longrightarrow C_{0}^{\infty}\left(\mathbb{R}^{n}, \mathbb{R}^{p}\right)
$$

of $f$ relative to $\mathscr{C}$ is given by

$$
T_{f}(\xi)=d f \circ \xi+\xi \circ f .
$$

It is clear that $\theta(n)$ is a $C_{0}^{\infty}\left(\boldsymbol{R}^{n}\right)$-module, but $T_{f}$ is not a $C_{0}^{\infty}\left(\mathbb{R}^{n}\right)$-homomorphism. Hence, $\left(C_{0}^{\infty}\left(\boldsymbol{R}^{n}, \boldsymbol{R}^{n}\right), \mathscr{C}(n), C_{0}^{\infty}\left(\boldsymbol{R}^{n}\right)\right)$ is not essential.

In fact, there exists the example for which the versality theorem cannot hold. (Belitskii [1].)

A-3) $\quad E(M)=T(M)$.

In this case, $\Gamma_{T}^{\infty}(n)$ is the set of vector field germs on $\mathbb{R}^{n}$ at the origin. The most natural equivalence relation between germs of vector fields is the smooth equivalence. 
If we let $\mathscr{G}_{T}(M)=\{(\phi, d \phi) \mid \phi$ is a local diffeomorphism on $M\}$, then the $\mathscr{G}_{T}$-equivalence is the smooth equivalence between vector field germs.

For any $\xi \in \Gamma_{T}^{\infty}(n)=\theta(n)$, the infinitesimal map

$$
T_{\xi}: \theta(n) \longrightarrow C_{0}^{\infty}\left(\boldsymbol{R}^{n}, T_{0} \mathbb{R}^{n}\right)
$$

is given by Lie derivative:

$$
T_{\xi}(\eta)=L_{\eta} \xi
$$

Since Lie derivative is not a $C_{0}^{\infty}\left(\mathbb{R}^{n}\right)$-homomorphism, then $\left(\Gamma_{T}^{\infty}(n), \mathscr{G}_{T}(n)\right.$, $C_{0}^{\infty}\left(\boldsymbol{R}^{n}\right)$ ) is not essential.

In fact, it is known that the versality theorem does not hold for the category of unfoldings of vector field germs relative to smooth equivalence. (See [1].)

A-4) $E(M)=\wedge_{p} T^{*}(M)$.

In this case, $\Gamma_{E}^{\infty}(n)=\Omega^{p}(n)$ is the set of germs of differential $p$-forms.

Let $\mathscr{G}_{\Omega}(M)=\left\{\left(\phi, \wedge^{t} d \phi\right) \mid \phi\right.$ is a local diffeomorphism on $\left.M\right\}$, then the $\mathscr{G}_{\Omega^{2}}$-equivalence on $\Omega^{p}(n)$ is the smooth equivalence between germs of differential $p$-forms.

By the same reason as the case of vector field germs, $\left(\Omega^{p}(n), \mathscr{G}_{\Omega}(n), C_{0}^{\infty}\left(\mathbb{R}^{n}\right)\right)$ is not essential.

But, we have not known the example for which the versality theorem cannot hold. It is conjectured that there exists the example of differential form for which the versality theorem cannot hold.

A-5) $\quad E(M)=\underset{k}{\oplus} T^{*}(M) \quad(k<\operatorname{dim}(M))$.

In this case, $\operatorname{Pf}_{k}(M)=\Gamma_{E}^{\infty}(M)$ is the set of local Pfaffian systems.

For any $\omega=\left(\omega_{1}, \ldots, \omega_{k}\right) \in \mathrm{Pf}_{k}(M)$, there exists the local coordinate system $\left(U,\left(x_{1}, \ldots, x_{n}\right)\right)$ and smooth functions $a_{i}^{j}: U \rightarrow \mathbb{R}(i=1, \ldots, k ; j=1, \ldots, n)$ such that

$$
\omega_{i}\left(x_{1}, \ldots, x_{n}\right)=\sum_{j=1}^{n} a_{i}^{j}(x) d x_{j} .
$$

Definition 4.3. We say that $x_{0} \in U$ is a singular point of $\omega$ if $\operatorname{rank}\left(a_{i}^{j}\left(x_{0}\right)\right)$ $<k$.

The most natural equivalence relation between germs of Pfaffian systems is the equivalence relation which is given by coordinate transformations on $M$. The same reason as the case $\mathrm{A}-4$ ), this equivalence relation cannot make the essential triple.

But, if only pay attention to singularities of Pfaffian systems, it is enough to 
consider the equivalence relation which preserve rank of $\left(a_{i}^{j}(x)\right)$ and make the essential triple.

One of the candidate of such an equivalence relation is given by the following essential pseudo-group:

$\mathscr{P}(M)=\left\{\left(h^{-1}, \underset{k}{\oplus} H^{*}\right) \mid H\right.$ is a local vector bundle automorphism on $T M$ which covers $h\}$.

A-6) For any vector bundle $E(M)$, let

$$
D: \Gamma_{E}^{\infty}(n) \longrightarrow \Gamma_{E}^{\infty}(n)
$$

be a linear partially differential operator.

Let $\Gamma_{E}^{D}(n)^{\omega}=D^{-1}(\omega)$ for $\omega \in \Gamma_{E}^{\infty}(n)$, then $C_{0}^{D}\left(\mathbb{R}^{n}, V\left(\mathbb{R}^{n}\right)\right)=D^{-1}(0)$.

Generators of $C_{0}^{\infty}\left(\mathbb{R}^{n}, V\left(\mathbb{R}^{n}\right)\right)$ over $C_{0}^{\infty}\left(\mathbb{R}^{n}\right)$ are given by $e_{i}: \mathbb{R}^{n} \rightarrow V\left(\mathbb{R}^{n}\right)$ $\left(i=1, \ldots, \operatorname{dim}\left(V\left(\mathbb{R}^{n}\right)\right)\right)$ which are defined by $e_{i}(x)=(0, \ldots, 0, \stackrel{i}{1}, 0, \ldots, 0)$ for any $x \in\left(\mathbb{R}^{n}, 0\right)$. Since $e_{\imath} \in C_{0}^{D}\left(\mathbb{R}^{n}, V\left(\mathbb{R}^{n}\right)\right)$ for any $i$, then

$$
\left\langle C_{0}^{D}\left(\mathbb{R}^{n}, V\left(\mathbb{R}^{n}\right)\right)\right\rangle_{C_{0}^{\infty}\left(\mathbb{R}^{n}\right)}=C_{0}^{\infty}\left(\mathbb{R}^{n}, V\left(\mathbb{R}^{n}\right)\right) .
$$

Hence, for the essential pseudo-group $\mathscr{G}_{E}(M)$ which satisfies conditions in Definition 1.11, the following versality theorem holds.

Theorem 4.4. Let $\sigma \in \Gamma_{E}^{D}(n)_{0}^{\omega}$ such that

$$
\operatorname{dim}_{\boldsymbol{R}} C_{0}^{\infty}\left(\mathbb{R}^{n} V,\left(\mathbb{R}^{n}\right)\right) /\left(C_{0}^{D}\left(\mathbb{R}^{n}, V\left(\mathbb{R}^{n}\right)\right)+T_{\sigma}^{\mathscr{G}}\left(\theta_{E}^{\mathscr{S}}(n)\right)\right)<+\infty .
$$

Let $\Sigma:\left(\mathbb{R}^{n} \times \mathbb{R}^{r},(0,0)\right) \rightarrow\left(E\left(\mathbb{R}^{n}\right), 0\right)$ be a D-unfolding of $\sigma$. If $C_{0}^{D}\left(\mathbb{R}^{n}, V\left(\mathbb{R}^{n}\right)\right) \subset T_{\sigma}^{\mathscr{g}}\left(\theta_{E}^{g}(n)\right)+V_{\Sigma}$, then $\Sigma$ is versal $D$-unfolding of $\sigma$ relative to $\mathscr{G}_{E}$.

For example, let $E(M)=M \times \mathbb{R}$ and $D=\Delta$ be a Laplace operator (i.e. $\left.\Delta=\partial^{2} / \partial x_{1}^{2}+\cdots+\partial^{2} / \partial x_{n}^{2}\right)$, then $\Gamma_{E}^{\Delta}(n)^{g}$ is the set of germs $u \in C_{0}^{\infty}\left(\mathbb{R}^{n}\right)$ which are solutions of the Poisson's equation $\Delta u=g$. In this case, $C_{0}^{\Delta}\left(\mathbb{R}^{n}\right)=\pi_{V}\left(\Delta^{-1}(0)\right)$ is the set of harmonic function germs at the origin.

Theorem 4.5. Let $f$ be a germ of solutions of Poisson's equation $\Delta f=g$ such that $\operatorname{dim}_{\mathbb{R}} C_{0}^{\infty}\left(\mathbb{R}^{n}\right) / J(f)<+\infty$. Let $F$ be a $\Delta$-unfolding of $f$. If $C_{0}^{\Delta}\left(\mathbb{R}^{n}\right)$ $\subset J(f)+V_{F}$, then $F$ is a versal $\Delta$-unfolding of $f$ relative to $\mathscr{R}$.

When $g=0$, then the above theorem seems to be applied for the qualitative study of phenomenons which are described by Harmonic function's potentials.

B) The case $R(n)=C_{0}^{G}\left(\mathbb{R}^{n}\right)$.

Here, let $G$ be a compact Lie group which acts linearly on $\mathbb{R}^{n}$ and $C_{0}^{G}\left(\mathbb{R}^{n}\right)$ be the set of germs of smooth $G$-invariant functions at the origin. 
B-1) $\quad E(M)=M \times \boldsymbol{R}^{p} \quad(M: G$-manifold $)$.

B-1-a) We let

$\mathscr{G}_{E}(M)=\mathscr{R}_{G}(M)=\{(h, h \times L) \mid h$ is a "local" equivariant diffeomorphism of $M$ and $L$ is a parallel transformation. .

(Here, $G$ acts on $\boldsymbol{R}^{p}$ trivially).

Let $\Gamma_{E}^{A}(n)$ be the set of $G$-equivariant section germs of $E\left(\boldsymbol{R}^{n}\right)$, then it is the set of $G$-invariant map germs $\left(\boldsymbol{R}^{n}, 0\right) \rightarrow \boldsymbol{R}^{p}$. We write it $C_{0}^{G}\left(\boldsymbol{R}^{n}, \boldsymbol{R}^{p}\right)$. In this case, the $\mathscr{R}_{G}$-equivalence on $C_{0}^{G}\left(\boldsymbol{R}^{n}, \boldsymbol{R}^{p}\right)$ is the $G$-right equivalence between $G$ invariant map germs.

The infinitesimal map

$$
T_{f}: \theta_{G}(n) \longrightarrow C_{0}^{G}\left(\boldsymbol{R}^{n}, \boldsymbol{R}^{p}\right)
$$

of $f$ relative to $\mathscr{R}_{G}$ is given by

$$
T_{f}(\xi)=d f \circ \xi,
$$

where $\theta_{G}(n)=\left\{\xi \in \theta(n) \mid \xi(g x)=T g \circ \xi(x)\right.$ for any $x \in\left(\boldsymbol{R}^{n}, 0\right)$ and $\left.g \in G\right\}$.

By the theorem of Schwartz $([7]), C_{0}^{G}\left(\boldsymbol{R}^{n}\right)$ is the sub $\boldsymbol{R}$-algebra of $C_{0}^{\infty}\left(\boldsymbol{R}^{n}\right)$ of finite type. Since $C_{0}^{G}\left(\boldsymbol{R}^{n}, \boldsymbol{R}^{p}\right) \cong C_{0}^{G}\left(\boldsymbol{R}^{n}\right) \times C_{0}^{G}\left(\boldsymbol{R}^{n}\right) \times \cdots \times C_{0}^{G}\left(\boldsymbol{R}^{n}\right)$, then $\left(C_{0}^{G}\left(\boldsymbol{R}^{n}\right.\right.$, $\left.\left.\boldsymbol{R}^{p}\right), \mathscr{R}_{G}(n), C_{0}^{G}\left(\boldsymbol{R}^{n}\right)\right)$ is essential.

If we denote the set $T_{f}\left(\theta_{E}(n)\right)$ by $J_{G}(f)$, then we have the following theorem.

Theorem 4.6. (Poénaru [6]). Let $F$ be a G-unfolding of $f \in C_{0}^{G}\left(\boldsymbol{R}^{n}, \boldsymbol{R}^{p}\right)$.

If $C_{0}^{G}\left(\boldsymbol{R}^{n}, \boldsymbol{R}^{p}\right)=J_{G}(f)+V_{F}$, then $F$ is a versal $G$-unfolding of $f$ relative to $\mathscr{R}_{G}$.

B-1-b) $\mathscr{G}_{E}(M)=\mathscr{A}_{u} t_{G}^{V}(M)=\{(h, H) \mid H$ is a "local" $G$-vector bundle automorphism on $M \times \boldsymbol{R}^{p}$ which covers h.\}. (Here, $G$ acts on $\boldsymbol{R}^{p}$ linearly).

Let $\boldsymbol{R}^{n}$ be a $G$-vector space. Let $\Gamma_{E}^{A}(n)$ be the set of $G$-equivariant section germs at the origin, then it is the set of $G$-equivariant map germs: $\left(\boldsymbol{R}^{n}, 0\right) \rightarrow \boldsymbol{R}^{p}$. We write it $C_{G}^{\infty}\left(\boldsymbol{R}^{n}, \boldsymbol{R}^{p}\right)$.

In this case, the $\mathscr{G}_{E}$-equivalence on $C_{G}^{\infty}\left(\boldsymbol{R}^{n}, \boldsymbol{R}^{p}\right)$ is the equivariant $\mathscr{K}$ equivalence between $G$-equivariant map germs. By this reason, we denote $\mathscr{A}_{\text {ret }}^{V}{ }_{G}^{V}(n)=\mathscr{K}_{G}(n, p)$.

Proposition 4.7. $C_{G}^{\infty}\left(\boldsymbol{R}^{n}, \boldsymbol{R}^{p}\right)$ is a finitely generated $C_{0}^{G}\left(\boldsymbol{R}^{n}\right)$-module.

Proof. Let $\left(\boldsymbol{R}^{p}\right)^{*}$ be a dual of $\boldsymbol{R}^{p}, G$ also acts on $\left(\boldsymbol{R}^{p}\right)^{*}$ linearly, for $(g \cdot w)(v)$ $=w\left(g^{-1} v\right)$ for any $w \in\left(\boldsymbol{R}^{p}\right)^{*}$ and $v \in \boldsymbol{R}^{p}$. We consider the diagonal action on $\boldsymbol{R}^{n} \times\left(\boldsymbol{R}^{p}\right)^{*}$. 
If $f \in C_{G}^{\infty}\left(\boldsymbol{R}^{n}, \boldsymbol{R}^{p}\right)$, it induces $f^{\prime} \in C_{(0,0)}^{G}\left(\mathbb{R}^{n} \times\left(\boldsymbol{R}^{p}\right)^{*}\right)$ by $f^{\prime}(v, w)=w(f(v))$. Since $C_{(0,0)}^{G}\left(\mathbb{R}^{n} \times\left(\boldsymbol{R}^{p}\right)^{*}\right)$ is the subring of $C_{(0,0)}^{\infty}\left(\mathbb{R}^{n} \times\left(\mathbb{R}^{p}\right)^{*}\right)$ of finite type (see [7]), there exist $p_{1}, \ldots, p_{k} \in C_{(0,0)}^{G}\left(\boldsymbol{R}^{n} \times\left(\boldsymbol{R}^{p}\right)^{*}\right)$ such that $P^{*}\left(C_{a}^{\infty}\left(\boldsymbol{R}^{k}\right)\right)=C_{(0,0)}^{G}$. $\left(\boldsymbol{R}^{n} \times\left(\boldsymbol{R}^{p}\right)^{*}\right)$. Then, we have $f^{\prime}(v, w)=H\left(p_{1}(v, w), \ldots, p_{k}(v, w)\right)$ for some $H \in$ $C_{a}^{\infty}\left(\mathbb{R}^{k}\right)$.

Since $f^{\prime}$ are linear for $w$, we have the following:

$$
f^{\prime}(v, w)=\sum_{i=1}^{k} \partial_{i}(H)_{P(v, v)} \partial_{w}\left(p_{i}\right)_{(v, 0)}(w),
$$

where $\partial_{i}$ denotes the derivative associated to $i$-th variable.

We can consider $\partial_{w^{\prime}}\left(p_{i}\right)_{(x, 0)}(w)$ as elements of $C_{G}^{\infty}\left(\mathbb{R}^{n}, \mathbb{R}^{p}\right)$. This completes the proof.

Q.E.D.

It is clear that $\left(C_{G}^{\circ}\left(\boldsymbol{R}^{n}, \boldsymbol{R}^{p}\right), \mathscr{K}_{G}(n, p), C_{0}^{G}\left(\boldsymbol{R}^{n}\right)\right)$ is essential.

Theorem 4.7. Let $F$ be a G-unfolding of an equivariant map germ $f:\left(\boldsymbol{R}^{n}, 0\right) \rightarrow\left(\boldsymbol{R}^{p}, 0\right)$.

If $C_{G}^{\infty}\left(\mathbb{R}^{n}, \mathbb{R}^{p}\right)=J_{G}(f)+f^{*}\left(1 i_{p}\right) C_{0}^{\infty}\left(\boldsymbol{R}^{n}, \mathbb{R}^{p}\right) \cap C_{0}^{G}\left(\mathbb{R}^{n}, \mathbb{R}^{p}\right)+V_{F}$, then $F$ is a versal G-unfolding relative to $\mathscr{K}_{G}$.

C) The case $R(n)=\mathbf{R}$.

In all above case, if we take finite jet spaces, then the versality theorems always hold.

\section{References}

[1] Belitskii, G. R., Equivalence and normal forms of germs of smooth mappings, Russian Math. Surveys, 33 (1978), 107-177.

[2] Golubitsky, M., Schaeffer, D., A theory for imperfect bifurcation via singularity theory, Commun. Pure Appl. Math., 32 (1979), 21-98.

[ 3 ] Martinet, J., Deploiments versels des applications différentiables er classification des applications stables in Singularités d'Applications Différentiable, Lecture Notes in Math., 535, Springer-Verlag, (1976), 1-44.

[4] Mather, J., Right equivalence, Unpublished Notes.

[5] - Stability of $C^{\infty}$ mappings III; Finitely determined map germs, Publ. Math. I. H. E. S., 35 (1968), 127-156.

[6] Poénaru, V., Singularités $C^{\infty}$ en présence de symétrie, Lecture Notes in Math., 510, Springer-Verlag, 1976.

[7] Schwarz, G., Smooth functions invariant under the action of a compact Lie group, Topology, 14 (1975), 63-68.

[8] Zakalyukin, V. M.. The versality theorem, Functional Anal. Appl., 7 (1973), 110-112. 
